\title{
SLAS Discovery
}

\section{SLC6A14, a pivotal actor on cancer stage: when function meets structure}

\begin{tabular}{|c|c|}
\hline Journal: & SLAS Discovery \\
\hline Manuscript ID & Draft \\
\hline Manuscript Type: & Original Research \\
\hline $\begin{array}{l}\text { Date Submitted by the } \\
\text { Author: }\end{array}$ & $\mathrm{n} / \mathrm{a}$ \\
\hline Complete List of Authors: & $\begin{array}{l}\text { Palazzolo, Luca; Università degli Studi di Milano, Dipartimento di Scienze } \\
\text { Farmacologiche e Biomolecolari } \\
\text { Parravicini, Chiara; Università degli Studi di Milano, Dipartimento di } \\
\text { Scienze Farmacologiche e Biomolecolari } \\
\text { Laurenzi, Tommaso; Università degli Studi di Milano, Dipartimento di } \\
\text { Scienze Farmacologiche e Biomolecolari } \\
\text { Adobati, Sara; Università degli Studi di Milano, Dipartimento di Scienze } \\
\text { Farmacologiche e Biomolecolari } \\
\text { Saporiti, Simona; Università degli Studi di Milano, Dipartimento di } \\
\text { Scienze Farmacologiche e Biomolecolari } \\
\text { Guerrini, Uliano; Università degli Studi di Milano, Dipartimento di Scienze } \\
\text { Farmacologiche e Biomolecolari } \\
\text { Gianazza, Elisabetta; Università degli Studi di Milano, Dipartimento di } \\
\text { Scienze Farmacologiche e Biomolecolari } \\
\text { Indiveri, Cesare; Università della Calabria, Dipartimento di Biologia, } \\
\text { Ecologia e Scienze della Terra } \\
\text { Anderson, Catriona; Newcastle University Institute for Cell and Molecular } \\
\text { Biosciences, Faculty of Medical Sciences } \\
\text { Thwaites, David; Newcastle University Institute for Cell and Molecular } \\
\text { Biosciences, Faculty of Medical Sciences } \\
\text { Eberini, Ivano; Università degli Studi di Milano, Dipartimento di Scienze } \\
\text { Farmacologiche e Biomolecolari }\end{array}$ \\
\hline Keywords: & $\begin{array}{l}\text { Computational chemistry, Pharmacology: ligand binding, receptor } \\
\text { binding, amino acid transporter, molecular docking, molecular modelling }\end{array}$ \\
\hline Abstract: & $\begin{array}{l}\text { ATB0, + (SLC6A14) is a sodium- and chloride-dependent neutral and } \\
\text { dibasic amino acid transporter that regulates the distribution of amino } \\
\text { acids across cell membranes. SLC6A14 is ubiquitous. The transporter is } \\
\text { over-expressed in many human cancers characterized by an increased } \\
\text { demand for amino acids; as such, it was recently acknowledged as a } \\
\text { novel target for cancer therapy. The knowledge on the molecular } \\
\text { mechanism of SLC6A14 transport is still limited, but some elegant } \\
\text { studies on related transporters report the involvement in the transport } \\
\text { mechanism of the symmetry of the } 12 \text { transmembrane a-helices, and } \\
\text { describe structural rearrangements mediated by electrostatic interactions } \\
\text { with some pivotal gating residues. } \\
\text { In the present work, we constructed a SLC6A14 model in outward facing } \\
\text { conformation via homology modelling and tested through molecular }\end{array}$ \\
\hline
\end{tabular}


dynamic simulation to predict amino acid residues critical for substrate recognition and translocation. We then docked the proteinogenic amino acids and other known substrates in the SLC6A14 binding site to study both gating regions and the exposed residues involved in transport. Interestingly, some of these residues correspond to those previously hypothesized by literature; however, we could identify also a novel relevant residue with such function

For the first time, by combined approaches of molecular docking and molecular dynamic simulations, we demonstrated the crucial role of these residues in neutral amino acids transport. This novel information unravels new aspects of human SLC6A14 structure/function relationship and may have also important outcomes in cancer through the design of novel inhibitors of SLC6A14-mediated transport.

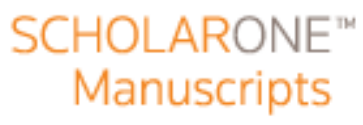




\title{
SLC6A14, a pivotal actor on cancer stage: when function meets structure
}

Luca Palazzolo ${ }^{1}$, Chiara Paravicini ${ }^{1}$, Tommaso Laurenzi ${ }^{1}$, Sara Adobati ${ }^{1}$, Simona Saporiti $^{1}$, Uliano Guerrini ${ }^{1}$, Elisabetta Gianazza ${ }^{1}$, Cesare Indiveri ${ }^{2}$, Catriona M.H. Anderson ${ }^{3}$, David T. Thwaites ${ }^{3}$, Ivano Eberini ${ }^{4,{ }^{*}}$

1 Dipartimento di Scienze Farmacologiche e Biomolecolari, Università degli Studi di Milano, Milano, Italy

2 Dipartimento di Biologia, Ecologia e Scienze della Terra, Università della Calabria, Cosenza, Italy

3 Institute for Cell \& Molecular Biosciences, Faculty of Medical Sciences, Framlington Place, Newcastle University, Newcastle upon Tyne, NE2 4HH, UK

4 Dipartimento di Scienze Farmacologiche e Biomolecolari \& DSRC, Università degli Studi di Milano, Milano, Italy

Corresponding author: Ivano Eberini, ivano.eberini@unimi.it

\begin{abstract}
$\mathrm{ATB}^{0,+}$ (SLC6A14) is a sodium- and chloride-dependent neutral and dibasic amino acid transporter that regulates the distribution of amino acids across cell membranes. SLC6A14 is ubiquitous. The transporter is over-expressed in many human cancers characterized by an increased demand for amino acids; as such, it was recently acknowledged as a novel target for cancer therapy. The knowledge on the molecular mechanism of SLC6A14 transport is still limited, but some elegant studies on related transporters report the involvement in the transport mechanism of the symmetry of the 12 transmembrane $\alpha$-helices, and describe structural rearrangements mediated by electrostatic interactions with some pivotal gating residues.

In the present work, we constructed a SLC6A14 model in outward facing conformation via homology modelling and tested through molecular dynamic simulation to predict amino acid residues critical for substrate recognition and translocation. We then docked the proteinogenic amino acids and other known substrates in the SLC6A14 binding site to study both gating regions and the exposed residues involved in transport. Interestingly, some of these residues correspond to those previously hypothesized by literature; however, we could identify also a novel relevant residue with such function
\end{abstract}

For the first time, by combined approaches of molecular docking and molecular dynamic simulations, we demonstrated the crucial role of these residues in neutral amino acids transport. This novel information unravels new aspects of human SLC6A14 structure/function relationship and may have also important outcomes in cancer through the design of novel inhibitors of SLC6A14-mediated transport. 


\section{Introduction}

Amino acids are the building blocks of protein synthesis (Sikder et al. 2018). They are essential components of all cells and are required for many metabolic pathways, nitrogen metabolism, neurotransmission and cell growth (Sikder et al. 2018; Scalise et al. 2016). Due to their physicochemical properties, amino acids cannot pass freely across cell membranes but rather undergo carrier-mediated transmembrane movement by specific amino acid transporters. Mammalian cells express numerous amino acid transporters in their plasma membranes to enable intake and efflux of amino acids between each cell and the extracellular environment. Most amino acid transporters have a relatively narrow substrate specificity. However, there is one mammalian amino acid transporter that has an unusually broad substrate selectivity transporting 18 of the 20 proteinogenic amino acids (Sikder et al. 2018). This transporter was originally characterized in rabbit ileum and named the $\beta$-alanine carrier although it also transported a wide range of zwitterionic and dibasic amino acids (Munck and Schultz 1969). Separately, a broad-scope amino acid transporter was identified in preimplantation mouse blastocysts and named System $B^{0,+}$ ((Van Winkle, Christensen, and Campione 1985). The transporter cDNA was cloned from human mammary gland and named $\mathrm{ATB}^{0,+}$ (Sloan and Mager 1999). $\mathrm{ATB}^{0,+}$ is a sodium- and chloride-dependent neutral and dibasic amino acid transporter which possesses all of the functional characteristics of both System $\mathrm{B}^{0,+}$ (Sloan and Mager 1999) and the $\beta$-alanine carrier (Anderson, Ganapathy, and Thwaites 2008) demonstrating that the early functional studies in intestine and blastocyst represent function of the same transport mechanism.

Mammalian membrane transporters are categorized (based upon amino acid sequence identity) into SoLute Carrier (SLC) familes (Hediger et al. 2013). ATB ${ }^{0,+}$ is the $14^{\text {th }}$ member of the SLC6 family and is also known as SLC6A14 (Bröer and Gether 2012). Although members of the SLC6 family show high levels of sequence identity between transporters, this family includes transporters with very different substrates where some are selective for amino acids and others are able to transport biogenic amines such as norepinephrine (NET/SLC6A2), dopamine (DAT/SLC6A3) or serotonin (SERT/SLC6A4) (Bröer and Gether 2012). The Transporter Classification Database (TCDB) of eukaryote and prokaryote transporters (Saier 2005), catalogues the human SLC6 transporters in the Neurotransmitter:Sodium Symporter family (NSS, TCDB family 2.A.22) which is within the broader Amino acid Polyamine organoCation (APC) Superfamily (Vastermark et al. 2014).

$\mathrm{ATB}^{0,+}$ couples cotransport of $2 \mathrm{Na}^{+}, 1 \mathrm{Cl}^{-}$and 1 amino acid, and is a highly concentrative electrogenic transport system with inward transmembrane amino acid transport being driven by membrane potential and $\mathrm{Na}^{+}$ and $\mathrm{Cl}^{-}$gradients (Sikder et al. 2018). $\mathrm{ATB}^{0,+}$ transports neutral amino acids and the dibasic amino acids arginine and lysine and thus transports all essential amino acids and only excludes the anionic amino acids glutamate and aspartate (Sikder et al. 2018). This broad substrate specificity also makes the ATB $^{0,+}$ transporter accessible to a wide variety of amino acid-based drugs and prodrugs including valacyclovir (Hatanaka 2004), valganciclovir (Umapathy, Ganapathy, and Ganapathy 2004), 1-methyltryptophan (Karunakaran et al. 2008), nitric oxide synthase inhibitors (Hatanaka et al. 2001) and carnitine and analogues (Nakanishi et al. 2001).

Abundant expression of $\mathrm{ATB}^{0,+}$ in healthy adult tissues is fairly restricted with expression mainly in retina, and respiratory and distal intestinal (ileum and colon) tissues where it is localized to luminal surfaces (Sikder et al. 2018; Hatanaka 2004; Ugawa et al. 2001). In contrast, $\mathrm{ATB}^{0,+}$ expression is upregulated in many solid tumours including colorectal (Gupta et al. 2005), cervical (Gupta et al. 2006), estrogen receptor-positive breast (Karunakaran et al. 2011) and pancreatic (Coothankandaswamy et al. 2016) cancers. The high expression in cancer tissues alongside the broad substrate specificity make $\mathrm{ATB}^{0,+}$ a good target for anti-cancer treatments using small molecules.

The structure of $\mathrm{ATB}^{0,+}$ is unknown but like most members of the APC superfamily (Vastermark et al. 2014) it is predicted to possess a LeuT-fold core structure, named after the amino acid transporter LeuT, the first transporter crystallised from the APC superfamily (Yamashita et al. 2005). Other APC superfamily members have since been crystallised and have broadly similar folds including the human serotonin transporter SERT (SLC6A4) (Coleman, Green, and Gouaux 2016) and the Drosophila melanogaster dopamine transporter DAT (SLC6A3) (Penmatsa, Wang, and Gouaux 2015). ATB $^{0,+}$ is predicted to have 12 transmembrane (TM) $\alpha$-helices. Based upon available APC superfamily crystal structures, TM 1-5 and TM 6-10 are predicted to be involved in the transport mechanism and have an antiparallel symmetry in their secondary structures giving the $\alpha$-helices a discrete mobility within the cell membrane and defining binding sites for transported substrates. The binding sites, S1 and S2, are located in the central binding pocket and in the extracellular vestibule, respectively (Kristensen et al. 2011). The central binding pocket must also contain sites that allow the binding of $2 \mathrm{Na}^{+}$and $1 \mathrm{Cl}^{-}$ions. TM 11 and 12 are homologous to structures required for the homo-dimerization of other transporters, but currently no evidence is 
available regarding $\mathrm{ATB}^{0,+}$ dimerization. For LeuT-fold proteins, a model of transport has been proposed (Gao et al. 2010): in the initial phase of the process, the protein lies in an "outward-facing" (OF) conformation and receives the transported substrate (and the ions) in binding site S2. The interaction between protein, ions and substrate promotes the translocation of transported solutes to the $\mathrm{S} 1$ binding site and closure of the gate. This process starts from the rotation of a specific conserved aromatic amino acid, which in turn leads to a conformational change in the whole protein, promoting its transition to an "inward-facing" (IF) conformation (Gao et al. 2010; Krammer et al. 2016). Afterwards, the substrate is released by the transporter in the intracellular space and the protein returns to the OF conformation, passing through intermediate transitory occluded states.

Therefore, the purpose of this investigation was to present for the first time the SLC6A14 3D structure, using homology modelling procedures to build a chimera model. Moreover, with this investigation, we meant: (i) to study in silico the structural features of the transporter, (ii) to identify in silico the key residues for the molecular recognition mechanism in the S1 binding site and (iii) to gather information about substrate binding.

\section{Materials and Methods}

\subsection{Comparative modelling of SLC6A14}

The sequence of human SLC6A14 (Uniprot ID: Q9UN76) was retrieved from the Uniprot knowledgebase database (Li et al. 2015). After a protein Blast (Li et al. 2015) search in the Protein Data Bank database for a human SLC6A14 homologue, the crystal structure of the Drosophila melanogaster sodium-dependent dopamine transporter (DAT) (PDB ID:4M48 ) (Penmatsa, Wang, and Gouaux 2015) was set as main template. A multiple alignment among the NSS family was produced with the Clustal Omega software (Larkin et al. 2007) and used for the homology modelling procedure. Since the crystal structure of DAT lacks the extracellular loop (EL) 2, a second local Blast search was set on residues S205-W270 of SLC6A14, identifying the crystallographic structure of phosphofructokinase (PFKA1) from Saccharomyces cerevisiae (PDB ID: 3080) (Banaszak et al. 2011) as an adequate template to fix the EL2 gap. The 3D structures of the two templates and of SLC6A14 were also investigated through both SWISS-MODEL and I-TASSER servers. Moreover, the transmembrane prediction programme TMHMM was used to determine the putative topology of SLC6A14, strenghtening the alignment between SLC6A14 and DAT from a structural point of view. PROTTER and was also used to build the prediction of secondary structure.

Selected 3D structures were first optimized and refined via further computational steps by correcting crystallographic-related errors through the MOE Structure Preparation Module of the Molecular Operating Environment 2018 (MOE, Chemical Computing Group, Montreal, Canada).

The selected portion (residues from L620 to R665) of phosphofructokinase template was carefully placed in the correspondence of DAT EL2. Three amino acids of both crystal structures were deleted at the point of connection between the two templates in order to have more flexibility to shape their junction in the chimeric model of SLC6A14, using the MOE Homology Model tool. Ten different intermediate models were built and submitted to energy minimization (EM) to release internal constraints. The top-scoring model, according to the GB/IV scoring function, was submitted to further EM with the 'FINE' option until the RMS gradient reached a value below 0.5 $\mathrm{kcal} / \mathrm{mol} / \AA$.

The Amber10:ETH force field with the reaction-field model for electrostatics was applied for the whole modelling procedure.

\subsection{Equilibration and cluster extrapolation}

Molecular dynamics (MD) simulation and frame clustering procedures were carried out with the Schrödinger Small-Molecule Drug Discovery Suite 2018-01 (D. E. Shaw Research, New York, NY, 2018, Schrödinger, New York, NY, 2018).

The Desmond System Builder tool was used to place the apo-model of SLC6A14 into a POPC membrane bilayer. Protein orientation was set up according to the OPM server (Lomize et al. 2012), which provides spatial arrangements of membrane proteins with respect to the hydrocarbon core of the lipid bilayer. The $\mathrm{N}$ - and $\mathrm{C}$ termini of the protein were capped. The system was solvated with 10,174 SPC water molecules in a cubic box with 90 Å edges; adding chloride ions neutralized the exceeding positive charge; sodium chloride was further added up to $0.15 \mathrm{M}$ concentration. The system was energy-minimized to relax the assembly and remove clashes between protein, membrane and solvent in the new setup. 
To produce an equilibrated model of SLC6A14, the system was submitted to a 500 ns molecular dynamic simulation (MD) using Desmond Molecular Dynamics tool. Periodic boundary conditions (PBC) and the following parameters were set: $300 \mathrm{~K}$ and Nose-Hoover thermostat for temperature coupling, 1 bar and Martyna-TobiasKlein piston for pressure coupling, 2 fs as integration time step. Coordinates and velocities of each atom were saved every 0.5 ps. The trajectory was then analysed using both Desmond Simulation Event Analysis tool and VMD (Phillips et al. 2005).

Desmond Trajectory Frame Clustering tool was used to cluster the whole 500 MD simulation in order to select the most representative frame (the centroid) for each cluster. Distances between clusters were computed from the RMSD matrix of alpha carbons with respect to the first frame of the MD simulation. Two different runs were performed to set the number of generable clusters according to the dendrogram.

The OPLS3e force field was applied both in the MD simulation and in the frame clusterization procedures.

\subsection{Docking}

The centroid of the most populated cluster was selected as reference structure for molecular docking procedures. Water, ions and the POPC membrane were washed out from the system and three pivotal ions $(1 \mathrm{Cl}$ and $2 \mathrm{Na})$ from the DAT template were transferred to the SLC6A14 equilibrated model. The ions::SLC6A14 3D structure was then energy-minimized using the Prime in order to fix structural issues in the ions binding domain.

Tested ligands were downloaded from PubChem and prepared for docking with the Schrödinger Ligand Preparation.

The molecular docking procedure was carried out with the Schrödinger Glide Docking in the "extra precision (XP)" mode in order to evaluate the ability of the tested ligands to bind the SLC6A14 binding channel, keeping only the five top-scoring poses.

The top-scoring solution for each ligand was submitted to the Schrödinger Prime MM-GBSA, which integrates molecular mechanics energies with the generalized Born and surface area continuum solvation (Genheden and Ryde 2015) in order to compute ligand binding and ligand strain energies for a set of ligands and a single receptor. The top-scoring solution for each ligand was also submitted to Desmond Molecular Dynamics tool for a 50 ns of MD simulation in order to study ligand::protein interaction and verify the stability of the ligand placement.

Periodic boundary conditions (PBC) and the following parameters were set for each MD simulation: $300 \mathrm{~K}$ and Nose-Hoover thermostat for temperature coupling, 1 bar and Martyna-Tobias-Klein piston for pressure coupling, $2 \mathrm{fs}$ as integration time step. Coordinates and velocities of each atom were saved every 0.5 ps. The trajectories were then analysed using Desmond Simulation Interaction Diagram tool and inspected through VMD. The OPLS3e force field was applied both in molecular docking procedures and in MD simulations.

\section{Results and Discussion}

\subsection{Secondary structure analysis and two-dimensional topology prediction}

None of the SLC6A14 homologous templates provides complete sequence coverage, as all of them leave out EL2. Not unexpectedly, the SWISS-MODEL and the I-TASSER predictions on the whole protein are unsatisfactory because, in both cases, the EL2 is predicted at an exceedingly high distance from a theoretical conformation resulting in very low local quality estimation. A very high number (>30) of outliers is observed in both Ramachandran plots. Supplementary Figure 1 reports the SWISS-MODEL quality estimation plot and the I-TASSER estimation distance plot.

Building a chimera model seems then to be the right strategy to study SLC6A14 at an atomistic level.

Taking the global alignment among members of the NSS family as the reference structure to model SLC6A14 in its OF conformation, we selected DAT (Penmatsa, Wang, and Gouaux 2015), with a sequence identity of $43 \%$ and a similarity of $59 \%$, as the most suitable template presently available. Moreover, unlike other suitable templates whose structure contains only one cation and one anion, DAT is co-crystallized with $2 \mathrm{Na}^{+}$ions and $1 \mathrm{Cl}^{-}$ion. On the other hand, according to BLAST, PFKA1, with a local sequence identity of $28 \%$ and local similarity of $46 \%$, appears to be the most suitable template for modelling EL2.

The second step towards building a chimera model of SLC6A14 was to compare its putative secondary structure and the two-dimensional topology predicted with three independent methods, while taking into account both the alignment and the topology of the DAT crystal (Penmatsa, Wang, and Gouaux 2015). Figure 1 shows the alignment among SLC6A14 and the selected templates; SLC6A14 is annotated according to TMHMM and PROTTER prediction. 
Figure 1

PROTTER and TMHMM prediction are compliant with the DAT secondary structure and its transmembrane placement. In particular, the first three TM $\alpha$-helices of DAT are more extended than expected from the prediction, while TM $\alpha$-helices from 4 to 7 and from 9 to 12 are in good agreement with the expectations. TM $\alpha$-helix 8 seems to be shifted towards the C-terminus by 5 amino acids with respect to PROTTER prediction, while it complies with TMHMM prediction. The alignment among SLC6A14, DAT and PFKA1 was carefully verified taking into account also the PROTTER and TMHMM prediction. Predicted topology of SLC6A14 is shown in Figure 2.

Figure 2

\subsection{Homology modelling of SLC6A14 and its MD equilibration}

The Ramachandran plot for the chimera model of SLC6A14, based on both DAT and PFKA1, shows only six outliers, none of them in the transmembrane domain (Supplementary Figure 2.A). Its secondary structure is compliant with both the DAT structure and the PROTTER and TMHMM predictions. As expected, SLC6A14 model has a LeuT-fold, with two well defined binding sites for both substrate/inhibitor and $\mathrm{Na}^{+} / \mathrm{Cl}^{-}$ions, respectively.

In order to equilibrate the chimera model of SLC6A14, we carried out a 500 ns MD simulation. The evaluation of the stability of our model during the simulation was based both on energetic and geometric parameters, as described in the following.

The general stability of the SLC6A14 model is confirmed by the tendency of the MD simulations to reach convergence. In detail, the tendency to reach an equilibrium state is suggested by the trend of the root mean square deviation (RMSD) values, which, after $100 \mathrm{~ns}$, reach a plateau around $3.5 \AA$ (Figure 3.A); its fluctuations towards the end of the simulation are around some thermal average structure. The lowest root mean square fluctuations (RMSF) are associated with $\alpha$-helices, the highest with the loops, both inter- and extra-cellular, with maximum in EL2 (Figure 3.B).

Figure 3

Protein secondary structure elements (SSE) i.e. $\alpha$-helices and $\beta$-strands, were monitored throughout the simulation. Figure 3.C reports SSE distribution by residue index, while Figure 4 summarizes the SSE composition for each trajectory frame over the course of the simulation and monitors each residue and its SSE assignment over time. Globally, the secondary structure of SLC6A14 is conserved during the MD simulation; the helices in EL2 are structured in over $50 \%$ of the frames, despite EL2 undergoing a strong rearrangement during the first 200 ns. In fact, EL2 migrates from a conformation extended towards the extracellular side to a more stable situation (Supplementary Figure 3), in which it establishes some H-bonds with the exposed amino acids of other ELs. Asn219 interacts with Lys225 via an H-bond, that is conserved for 70\% of the frames, shaping a turn-like structure and partially covering the SLC6A14 transport channel. This peculiar shape of EL2 is present in the frame selected for structural analysis, that is the centroid of the most populated cluster, composed by $70 \%$ of the all frames. The Ramachandran plot of the selected frame shows only one outlier (Supplementary Figure 2.B).

Figure 4

3.3 Structural features of the binding sites

Figure 5

To study the structural features of SLC6A14 transport channel, and its binding sites, we imported from DAT crystal structure to our SLC6A14 equilibrated model two $\mathrm{Na}^{+}$and one $\mathrm{Cl}^{-}$ions and, then, we relaxed the protein to avoid atom clashes. Figure 5 shows the SLC6A14 equilibrated structure after the local minimization of the ion pocket. 


\begin{abstract}
As mentioned in the Introduction, it is well known that, when in OF conformation, SLC6A family members have a binding site in the bottom of their transport channel. This binding site includes two specific regions: the first for the substrate, the second for the co-transported ions, which are a fundamental feature of all the SLC6 transporters.

In SLC6A14 we found two distinct sites in the S1 pocket that are unambiguously being occupied by a $\mathrm{Na}^{+}$ion, designated $\mathrm{Na} 1$ and $\mathrm{Na} 2$ (Singh 2008). Both $\mathrm{Na}^{+}$binding sites are believed to have a key role in stabilizing TM1 and TM6 in the presence of substrate.

$\mathrm{Na} 1$ binding site is closed towards the $\mathrm{Cl}$ binding site, and is divided from Na2 by TM1, sharing some amino acids with it. Na1 is not contiguous with the S1 substrate binding site, while the Na1 site insists on the S1 substrate binding site, and the $\mathrm{Na}^{+}$ion is available for interacting with the substrate. Residues composing the $\mathrm{Na} 1 \mathrm{and} \mathrm{Na} 2$ ion binding sites are conserved among the transporters of interest and, in general, are highly conserved across the whole SLC6 family (Kristensen et al. 2011). As suggested by Kristensen (Kristensen et al. 2011), the evidence of contiguous surfaces between $\mathrm{Na} 2$ and the substrate binding site can be the basis for a coupled translocation mechanism for both, $\mathrm{Na}^{+}$and substrate. On the other hand, the placement of $\mathrm{Cl}^{-}$in the $\mathrm{Na} 1$ binding site supports the idea that $\mathrm{Cl}^{-}$is translocated together with the $\mathrm{Na}^{+}$ion along the SLC6A14 channel during the transport of the substrate. The same ion placement has been reported also for the crystallographic structures of DAT (SLC6A3) and human SERT (SLC6A4) (Penmatsa, Wang, and Gouaux 2015; Coleman, Green, and Gouaux 2016). These data confirm that our equilibrated SLC6A14 model maintains its ion binding site (Figure 6.A).
\end{abstract}

\title{
Figure 6
}

The S1 substrate binding site is defined by polar, aromatic, and aliphatic amino acid side chains provided by all four TMs (TM1, TM3, TM6, and TM8) that surround the binding site, in addition to the backbone amide groups from the unwound regions of TM1 and TM6 (Singh 2008). The S1 pocket can be divided into two regions: a polar region formed exclusively by the unwound regions of TM1 and TM 6 and a hydrophobic pocket formed by aliphatic side chains from TM1, TM3, and TM6 (Figure 6.B).

In the SLC6A14 equilibrated model, we can also detect the S2 binding site in the gating region (Kristensen et al. 2011), which is partially defined at the bottom by three amino acids: Tyr321, Arg61and Asp478, which are partially conserved among SLC6A14, DAT and SLC6A4 (Figure 6.B).

Arg104 and Asp478 establish a H-bond, fixing the Arg104 in a conformation stretched toward the inside of the transport channel. Tyr321 is stretched toward the transport channel, unlike its corresponding amino acids Phe319 and Phe335 in DAT and SLC6A14, respectively.

From a structural point of view, this particular aromatic amino acid seems to assume the same function of Trp202, the gating residue of the arginine/agmatine antiporter AdiC. This gating mechanism is also supposed to be conserved for the SLC6 family (Pramod et al. 2013) and it was simulated for LAT1 (SLC7A5) via target molecular dynamics (Palazzolo et al. 2018). To evaluate the gating arrangement, we compared the placement of Phe319 in two DAT crystallographic structures in OF conformation, with gate open (Pdb ID: 4M48) (Penmatsa, Wang, and Gouaux 2015) and closed (Pdb ID: 4XPA) (Wang, Penmatsa, and Gouaux 2015), via a structural superposition. As shown in Figure 9.A, Tyr321 of the equilibrated model of SLC6A14 is in an intermediate position between DAT Phe319 open and closed conformations, suggesting that this amino acid has an intrinsic flexibility also in the absence of substrates/inhibitors. This intrinsic flexibility is also observed during the MD simulation, during which Tyr321 oscillates between open and partially closed conformation, with respect to DAT as a reference (Figure 7.A), suggesting that this amino acid has many degrees of freedom while it explores the conformational spaces in its surroundings.

Figure 7 
Moreover, we also identified the Trp327 as second putative inner gate of SLC6A14. In particular, this amino acid rotates from a DAT Phe325-like conformation to an occluded one, defining the bottom of the S1 substrate binding site. Evidence that Trp327 can be involved in the inner gating mechanism can be obtained from a structural analysis of the DAT Phe325 behaviour in presence of different inhibitors. As shown in Figure 7.B, DAT Phe325 has some degrees of freedom to coordinate itself with the transported molecules. In the two reference crystallographic structures of DAT, Phe325 is oriented by the inhibitors and it is alternately open or closed, with Phe319 behaving as the outer gate.

\subsection{Molecular docking}

As mentioned in the introduction, there are some aa transporters, among which SLC6A14, that transports 18 of the 20 amino acids, i.e. all of the proteogenic amino acids except glutamate and aspartate (Scalise et al. 2016). Molecular docking simulation was applied to place the substrates into the S1 binding site, appraising substrate::carrier interactions and computing the relevant docking scores. On the basis of these results, each complex substrate::carrier was submitted to a $50 \mathrm{~ns}$ MD simulation to evaluate the stability of the interactions and to assess the molecular recognition mechanisms, looking for pivotal amino acids involved in the recognition of the different substrates.

As expected, most of the tested ligands have docking scores ranging from $-8 \mathrm{kcal} / \mathrm{mol}$ to $-1 \mathrm{kcal} / \mathrm{mol}$, values that are compliant with solute transport. Supplementary Table 1 reports the most interesting interactions between tested compound and SLC6A14 amino acids during the MD simulations.

Tryptophan and 1-methyl-L-tryptophan are the substrates associated with the best docking scores, i.e. -7.5 $\mathrm{kcal} / \mathrm{mol}$ and $-6.3 \mathrm{kcal} / \mathrm{mol}$, respectively. These substrates are placed into the S1 binding site with overlapping docking poses (Figure 8); their interactions are mainly with Tyr321, Trp327, Phe320, Ser324, Leu56 and Ser423. The MD simulations show that the most conserved contacts between tryptophan and SLC6A14 amino acids are with Tyr52, Ala53, Gly57, Tyr132 and Tyr321 and that all of these contacts occur via H-bonds. On the other hand, 1-methyl-L-tryptophan interacts via H-bonds with Leu56, Gly57, Tyr132, Phe320 and Ser324, and all of these contacts are conserved during the MD simulation. Ala53 establishes an important ionic interaction with 1-methylL-tryptophan that is conserved in the whole MD simulation. Both substrates interact also with $\mathrm{Na}^{+}$in the $\mathrm{Na} 1$ ion binding site for $\sim 97 \%$ of the simulation total frames. These results are in agreement with those of both Hatanaka et al. (2004) and Karunakaran et al. (2008).

\section{Figure 8}

Tyrosine overlap the Tryptophan docking pose, with associated a docking score of $-5.4 \mathrm{kcal} / \mathrm{mol}$, mainly interacting with Ala326 and Ser324. The MD simulation highlight also H-bonds interaction with Val54, Leu56, Ser324 and Ser 423 and other important hydrophobic interactions with Tyr132, Tyr321, Trp327.

Arginine, Leucine and Isoleucine share the same binding pose and have similar docking scores of $-4.9 \mathrm{kcal} / \mathrm{mol}$, $4.6 \mathrm{kcal} / \mathrm{mol}$ and $-4.4 \mathrm{kcal} / \mathrm{mol}$, respectively. Arginine mainly interacts with Tyr132, Phe320 and Asp478, while Leucine and Isoleucine establishes H-bonds with Tyr132, Phe320, Val325 and Ser324. The MD simulations show that Asp478 is the pivotal binder for Arginine and it is probably involved also in the recognition mechanism, while for Leucine and IsoleucineTyr321 is the pivotal amino acid with which interacts for $~ 94 \%$ of the MD simulation. Arginine interacts with $\mathrm{Cl}^{-}$, while Leucine interacts with $\mathrm{Na}^{+}$in the $\mathrm{Na} 1$ ions binding site.

As reported in Scalise et al. (2016), Glutamine is compressed between Val128 and Tyr321, with a docking score value of $-3.8 \mathrm{kcal} / \mathrm{mol}$. Glutamine also interacts via H-bonds with Tyr132, Phe320 and Asp478 in its binding pose, while the MD simulation detects ionic interactions with Ala53, Tyr321 and Ser324. Glutamine also interacts with $\mathrm{Na}^{+}$in the $\mathrm{Na} 1$ ion binding site.

Proline and Cysteine have very similar both binding poses and docking scores of about $-3.1 \mathrm{kcal} / \mathrm{mol}$. This tested amino acids share the same interaction with Tyr132 and Phe320, establishing four H-bonds during the MD simulations also with Ser324 and Val325. Contact with Tyr132, Phe320 and Ser324 are conserved for both substrates for over $90 \%$ of the total time of the MD simulations. 


\section{Conclusions}

With our research, we propose, the first atomistic model of SLC6A14 transporter based on a chimeric approach: the two templates selected for modelling the OF SLC6A14 state are the most suitable so far available, and one of them has also been profitably used in a recent publication (Scalise et al. 2016). Thanks to 500 ns of MD simulation, we identified a specific behaviour of Tyr321, highlighting its role in the outer gating mechanism that is coordinated with the involvement of Arg104 and Asp478. We also identified Trp327 as putative inner gate, in analogy to DAT, our reference template. These two amino acids define the $\mathrm{S} 1$ binding site while Tyr321 is at the same time at the bottom of the S2 binding site. From the cluster analysis we selected a reference 3D structure of SLC6A14 and we observed that ions binding sites are conserved across the SLC6 family.

Natural amino acids and some other well-known substrates were tested for interaction via molecular docking simulation. For all of them both the docking scores were evaluated, and the molecular recognition mechanism was characterized, highlighting that both gates are essential for binding. In a broader way, Try52, Gly57, Val128, Ser322 and Ser324 compose an ensemble of amino acids that orient the substrates, confirming the structural findings of Yamashita et al. (2005), Scalise et al. (2016) and Edwards et al. (2018).

\section{Author contribution}

LP set up the homology modelling procedure, performed part of the simulations, conducted part of the analyses and drafted the manuscript. CP, SA, SS conducted part of the analyses. CP, TL and UG performed part of the simulations and contributed to the data analysis. $\mathrm{Cl}, \mathrm{CA}$, DT and IE conceived the concept of this work. Cl, CA, DT, EG and IE supervised the work and revised the manuscript.

\section{Funding}

This research was supported by grants from MIUR Progetto Eccellenza. IE gratefully acknowledges departmental Linea 2 - Azione A 2017 funding.

\section{References}

Anderson, Catriona M.H., Vadivel Ganapathy, and David T. Thwaites. 2008. "Human Solute Carrier SLC6A14 Is the $\beta$ Alanine Carrier." Journal of Physiology 586 (17): 4061-67. doi:10.1113/jphysiol.2008.154500.

Banaszak, Katarzyna, Ingrid Mechin, Galina Obmolova, Michael Oldham, Simon H. Chang, Teresa Ruiz, Michael 
Radermacher, Gerhard Kopperschläger, and Wojciech Rypniewski. 2011. "The Crystal Structures of Eukaryotic Phosphofructokinases from Baker's Yeast and Rabbit Skeletal Muscle." Journal of Molecular Biology. doi:10.1016/j.jmb.2011.01.019.

Bröer, Stefan, and Ulrik Gether. 2012. "The Solute Carrier 6 Family of Transporters." British Journal of Pharmacology. doi:10.1111/j.1476-5381.2012.01975.x.

Coleman, Jonathan A., Evan M. Green, and Eric Gouaux. 2016. "Thermostabilization, Expression, Purification, and Crystallization of the Human Serotonin Transporter Bound to $<$ em $>S</ E m>$-Citalopram." Journal of Visualized Experiments. doi:10.3791/54792.

Coothankandaswamy, V., S. Cao, Y. Xu, P. D. Prasad, P. K. Singh, C. P. Reynolds, S. Yang, J. Ogura, V. Ganapathy, and Y. D. Bhutia. 2016. "Amino Acid Transporter SLC6A14 Is a Novel and Effective Drug Target for Pancreatic Cancer." British Journal of Pharmacology 173 (23): 3292-3306. doi:10.1111/bph.13616.

Gao, Xiang, Lijun Zhou, Xuyao Jiao, Feiran Lu, Chuangye Yan, Xin Zeng, Jiawei Wang, and Yigong Shi. 2010. "Mechanism of Substrate Recognition and Transport by an Amino Acid Antiporter." Nature 463 (7282): 828-32. doi:10.1038/nature08741.

Genheden, Samuel, and UIf Ryde. 2015. "The MM/PBSA and MM/GBSA Methods to Estimate Ligand-Binding Affinities." Expert Opinion on Drug Discovery. doi:10.1517/17460441.2015.1032936.

Gupta, Naren, Seiji Miyauchi, Robert G. Martindale, Anne V. Herdman, Robert Podolsky, Katsuya Miyake, Sela Mager, Puttur D. Prasad, Malliga E. Ganapathy, and Vadivel Ganapathy. 2005. "Upregulation of the Amino Acid Transporter ATB0,+ (SLC6A14) in Colorectal Cancer and Metastasis in Humans." Biochimica et Biophysica Acta Molecular Basis of Disease. doi:10.1016/j.bbadis.2005.04.002.

Gupta, Naren, Puttur D. Prasad, Sharad Ghamande, Pamela Moore-Martin, Anne V. Herdman, Robert G. Martindale, Robert Podolsky, Sela Mager, Malliga E. Ganapathy, and Vadivel Ganapathy. 2006. "Up-Regulation of the Amino Acid Transporter ATB0,+ (SLC6A14) in Carcinoma of the Cervix." Gynecologic Oncology. doi:10.1016/j.ygyno.2005.08.016.

Hatanaka, T. 2004. "Transport of Amino Acid-Based Prodrugs by the Na+- and Cl--Coupled Amino Acid Transporter ATB0,+ and Expression of the Transporter in Tissues Amenable for Drug Delivery." Journal of Pharmacology and Experimental Therapeutics 308 (3): 1138-47. doi:10.1124/jpet.103.057109.

Hatanaka, T., T. Nakanishi, W. Huang, F. H. Leibach, P. D. Prasad, V. Ganapathy, and M. E. Ganapathy. 2001. "Na+- and Cl--Coupled Active Transport of Nitric Oxide Synthase Inhibitors via Amino Acid Transport System B0,+." Journal of Clinical Investigation 107 (8): 1035-43. doi:10.1172/JCI12060.

Hediger, Matthias A., Benjamin Clémençon, Robert E. Burrier, and Elspeth A. Bruford. 2013. "The ABCs of Membrane Transporters in Health and Disease (SLC Series): Introduction." Molecular Aspects of Medicine 34 (2-3): 95-107. doi:10.1016/j.mam.2012.12.009.

Karunakaran, Senthil, Sabarish Ramachandran, Veena Coothankandaswamy, Selvakumar Elangovan, Ellappan Babu, Sudharsan Periyasamy-Thandavan, Ashish Gurav, et al. 2011. "SLC6A14 (ATB 0,+) Protein, a Highly Concentrative and Broad Specific Amino Acid Transporter, Is a Novel and Effective Drug Target for Treatment of Estrogen Receptor-Positive Breast Cancer." Journal of Biological Chemistry. doi:10.1074/jbc.M111.229518.

Karunakaran, Senthil, Nagavedi S. Umapathy, Muthusamy Thangaraju, David H. Munn, Puttur D. Prasad, Vadivel Ganapathy, Takahiro Hatanaka, and Shiro Itagaki. 2008. "Interaction of Tryptophan Derivatives with SLC6A14 (ATB 0,+) Reveals the Potential of the Transporter as a Drug Target for Cancer Chemotherapy." Biochemical Journal 414 (3): 343-55. doi:10.1042/bj20080622.

Krammer, Eva Maria, Kassem Ghaddar, Bruno Andr??, and Martine Pr??vost. 2016. "Unveiling the Mechanism of Arginine Transport through AdiC with Molecular Dynamics Simulations: The Guiding Role of Aromatic Residues." PLOS ONE 11 (8): 1-29. doi:10.1371/journal.pone.0160219.

Kristensen, Anders S, Jacob Andersen, T. N. Jorgensen, L. Sorensen, Jacob Eriksen, Claus J Loland, K. Stromgaard, and U. Gether. 2011. "SLC6 Neurotransmitter Transporters: Structure, Function, and Regulation." Pharmacological Reviews. doi:10.1124/pr.108.000869.

Larkin, M. A., G. Blackshields, N. P. Brown, R. Chenna, P. A. Mcgettigan, H. McWilliam, F. Valentin, et al. 2007. “Clustal W and Clustal X Version 2.0." Bioinformatics 23 (21): 2947-48. doi:10.1093/bioinformatics/btm404.

Li, Weizhong, Andrew Cowley, Mahmut Uludag, Tamer Gur, Hamish McWilliam, Silvano Squizzato, Young Mi Park, Nicola Buso, and Rodrigo Lopez. 2015. "The EMBL-EBI Bioinformatics Web and Programmatic Tools Framework." Nucleic Acids Research 43 (W1): W580-84. doi:10.1093/nar/gkv279.

Lomize, Mikhail A., Irina D. Pogozheva, Hyeon Joo, Henry I. Mosberg, and Andrei L. Lomize. 2012. “OPM Database and PPM Web Server: Resources for Positioning of Proteins in Membranes." Nucleic Acids Research 40 (D1). doi:10.1093/nar/gkr703.

Munck, B. G., and Stanley G. Schultz. 1969. "Interactions between Leucine and Lysine Transport in Rabbit Ileum." BBA - Biomembranes. doi:10.1016/0005-2736(69)90142-4.

Nakanishi, Takeo, Takahiro Hatanaka, Wei Huang, Puttur D. Prasad, Frederick H. Leibach, Malliga E. Ganapathy, and 
Vadivel Ganapathy. 2001. "Na+ - and Cl- -Coupled Active Transport of Carnitine by the Amino Acid Transporter ATB0,+ from Mouse Colon Expressed in HRPE Cells and Xenopus Oocytes." Journal of Physiology 532 (2): $297-$ 304. doi:10.1111/j.1469-7793.2001.0297f.x.

Palazzolo, Luca, Chiara Parravicini, Tommaso Laurenzi, Uliano Guerrini, Cesare Indiveri, Elisabetta Gianazza, and Ivano Eberini. 2018. "In Silico Description of LAT1 Transport Mechanism at an Atomistic Level." Frontiers in Chemistry. doi:10.3389/fchem.2018.00350.

Penmatsa, Aravind, Kevin H. Wang, and Eric Gouaux. 2015. "X-Ray Structures of Drosophila Dopamine Transporter in Complex with Nisoxetine and Reboxetine." Nature Structural and Molecular Biology. doi:10.1038/nsmb.3029.

Phillips, James C., Rosemary Braun, Wei Wang, James Gumbart, Emad Tajkhorshid, Elizabeth Villa, Christophe Chipot, Robert D. Skeel, Laxmikant Kalé, and Klaus Schulten. 2005. "Scalable Molecular Dynamics with NAMD." Journal of Computational Chemistry. doi:10.1002/jcc.20289.

Pramod, Akula Bala, James Foster, Lucia Carvelli, and L. Keith Henry. 2013. "SLC6 Transporters: Structure, Function, Regulation, Disease Association and Therapeutics." Molecular Aspects of Medicine. doi:10.1016/j.mam.2012.07.002.

Saier, M. H. 2005. "TCDB: The Transporter Classification Database for Membrane Transport Protein Analyses and Information." Nucleic Acids Research. doi:10.1093/nar/gkj001.

Scalise, Mariafrancesca, Lorena Pochini, Michele Galluccio, and Cesare Indiveri. 2016. "Glutamine Transport. From Energy Supply to Sensing and Beyond." Biochimica et Biophysica Acta - Bioenergetics 1857 (8). Elsevier B.V.: 1147-57. doi:10.1016/j.bbabio.2016.03.006.

Sikder, Mohd Omar F., Shengping Yang, Vadivel Ganapathy, and Yangzom D. Bhutia. 2018. "The Na+/Cl--Coupled, Broad-Specific, Amino Acid Transporter SLC6A14 (ATB0,+): Emerging Roles in Multiple Diseases and Therapeutic Potential for Treatment and Diagnosis." The AAPS Journal 20 (1). The AAPS Journal: 12. doi:10.1208/s12248-0170164-7.

Singh, Satinder K. 2008. "LeuT: A Prokaryotic Stepping Stone on the Way to a Eukaryotic Neurotransmitter Transporter Structure." Channels. doi:10.4161/chan.2.5.6904.

Sloan, Jennifer L., and Sela Mager. 1999. "Cloning and Functional Expression of a Human Na+ and Cl--Dependent Neutral and Cationic Amino Acid Transporter B0+." Journal of Biological Chemistry 274 (34): 23740-45. doi:10.1074/jbc.274.34.23740.

Ugawa, Shinya, Yoko Sunouchi, Takashi Ueda, Eri Takahashi, Yoshitsugu Saishin, and Shoichi Shimada. 2001. "Characterization of a Mouse Colonic System B ${ }^{0+}$ Amino Acid Transporter Related to Amino Acid Absorption in Colon." American Journal of Physiology-Gastrointestinal and Liver Physiology. doi:10.1152/ajpgi.2001.281.2.G365.

Umapathy, Nagavedi S., Vadivel Ganapathy, and Malliga E. Ganapathy. 2004. "Transport of Amino Acid Esters and the Amino-Acid-Based Prodrug Valganciclovir by the Amino Acid Transporter ATB 0,+." Pharmaceutical Research. doi:10.1023/B:PHAM.0000033019.49737.28.

Van Winkle, L. J., H. N. Christensen, and A. L. Campione. 1985. "Na+-Dependent Transport of Basic, Zwitterionic, and Bicyclic Amino Acids by a Broad-Scope System in Mouse Blastocysts." Journal of Biological Chemistry 260 (22): 12118-23.

Vastermark, Ake, Simon Wollwage, Michael E. Houle, Rita Rio, and Milton H. Saier. 2014. "Expansion of the APC Superfamily of Secondary Carriers." Proteins: Structure, Function and Bioinformatics. doi:10.1002/prot.24643.

Wang, Kevin H., Aravind Penmatsa, and Eric Gouaux. 2015. "Neurotransmitter and Psychostimulant Recognition by the Dopamine Transporter." Nature. doi:10.1038/nature14431.

Yamashita, Atsuko, Satinder K. Singh, Toshimitsu Kawate, Yan Jin, and Eric Gouaux. 2005. "Crystal Structure of a Bacterial Homologue of $\mathrm{Na}+/ \mathrm{Cl}--$ Dependent Neurotransmitter Transporters." Nature 437 (7056): 215-23. doi:10.1038/nature03978.

Figure 1. Alignment among SLC6A14, DAT and PFKA1. TMHMM and PROTTER prediction are marked as violet bar, while secondary structure i.e. $\alpha$-helices and $\beta$-sheets of both DAT and PFKA1 are coloured in red and yellow, respectively.

Figure 2. 2D topology and transmembrane prediction for SLC6A14. Results with PROTTER are shown in (A), with TMHMM in (B). In (A) transmembrane helices are numbered from 1 to 12 and the putative N-linked glycosylation sites is marked in green. 
Figure 3: Stability of SLC6A14 model. (A) Root mean square deviation of SLC6A14 $\alpha$-carbon computed for each frame of MD simulation with respect to frame 0. (B) Root mean square fluctuation of SLC6A14 $\alpha$-carbon during the MD simulation. (C) Secondary structure elements: $\alpha$-helical regions are highlighted in red These regions are defined by helices that persist over $70 \%$ of the entire simulation.

Figure 4: Stability of SLC6A14 model. (Too) Root mean square deviation of SLC6A14 $\alpha$-carbons computed for each frame of MD simulation with respect to frame 0. (Bottom) Root mean square fluctuation of SLC6A14 $\alpha$-carbon during the MD simulation. (C) Secondary structure elements. $\alpha$-helical regions, defined as structures that persist over $70 \%$ of the entire simulation, are highlighted in red.

Figure 5. SLC6A14 equilibrated model. (A) Transversal view, (B) bottom and (C) top views. Secondary structure is represented by ribbons and coloured from $\mathrm{N}$ (blue) to $\mathrm{C}$ terminus (red); ions are represented as balloons.

Figure 6: SLC6A14 binding sites. (A) lons binding site, and (B) S1 and S2 substrate binding site surfaces in SLC6A14. Putative amino acids involved in the gating mechanism are outlined. The molecular surfaces of SLC6A14 binding sites computed as van der Waals interaction surface are represented with green, magenta and blue lines for hydrophobic, $\mathrm{H}$-bonding and mild polar interactions, respectively.

Figure 7: (A) Outer gate of SLC6A14 and its superposition to DAT crystallographic structures both in OF-open and OF-closed conformations. (B) SLC6A14 and DAT gates; as for other APCs, SLC6A14 seems to have two distinct aromatic amino acids that are involved in the gating mechanism. SLC6A14, DAT OF-open and DAT OF-closed are represented in yellow, light blue and light green, respectively.

Figure 8: (A) Tryptophan and 1-methyl-L-tryptophan, and (B) valaciclovir and valganciclovir binding poses. SLC6A14 helices and gating amino acids are coloured in yellow. 
TMHMM

S6A14_HUMAN 1 MDKLKCPSFFKCREKEKVSASSENFHVG---------ENDEN---QDRGNW

DAT_DROME

TMHMM

PROTTER

S6A14_HUMAN

DAT_DROME

TMHMM

PROTTER

S6A14_HUMAN

DAT_DROME

TMHMM

PROTTER

S6A14_HUMAN

DAT_DROME

PFK1_YEAST

1 ----------------MSP T GH I SKSKTP TP HDNDNNS I SDERETW

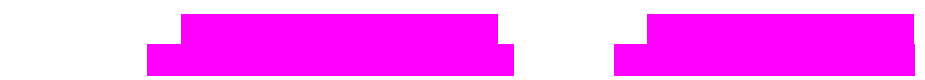

40 SKKSDYLLSMIGYAVGLGNVWRFPYLTYSNGGGAFLIPYAIMLALAGLPL 31 SGKVDFLLSVIGFAVDLANVWRFPYLCYKNGGGAFLVPYGIMLAVGGIPI

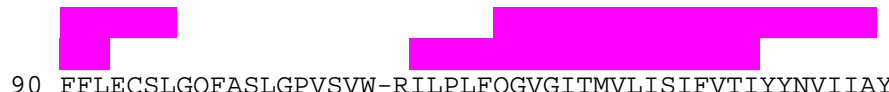

TMHMM

PROTTER

S6A14_HUMAN

DAT_DROME

PFK1_YEAST

TMHMM

PROTTER

S6A14_HUMAN

DAT_DROME

139 SLYYMFASFQSELPWKNCSS-WSDKNC--------SRSP IVTHCNVSTV131 SLRFFFASFTNSLPWT SCNNIWNTPNCRPFESQNASRVPVIG--NYSDLY $616-----(16)$

TMHMM PROTTER S6A14_HUMAN DAT_DROME

179 NKGIQEI IQMNK---SWVDINNF TC INGSEI----YQP GQLPSEQYWNK179 AMGNQSLLY---------NETYMNGSSLDTSAVGHVEGFQSAASEYFNRY 631 NGFSGLIQTGEVKELSWIDVENWHNLGGSEI----GTNR

TMHMM PROTTER S6A14_HUMAN DAT_DROME 221 -VALQRSSGMNETGVIVWYLALCLLLAWLIVGAALFKGIKSSGKVVYFTA 220 ILELNRSE G I HDLGA I KWDMALCLLIVYLICYF SLWKGISTSGKVVWFTA

270 LFPYVVLLILLVRGATLEGASKGISYYIGAQSNFTKLKEAEVWKDAATQI 270 LFPYAVLLILLIRGLTLPGSFLGIQYYI--TPNFSAIYKAEVWVDAATQV

TMHMM PROTTER S6A14_HUMAN DAT_DROME

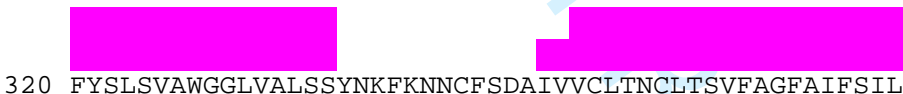

作 318 FFSIGP GFGVLLAYASYNKYHNNVYKDALLTSF INSATSF IAGFVIF SVI

TMHMM PROTTER S6A14_HUMAN DAT_DROME

TMHMM PROTTER S6A14_HUMAN DAT_DROME

370 GHMAHISGKEVSOVVKSGFDLAF IAYPEALAQLP GGPFWS ILFFFMLLTI 368 GYMAHTLGVRIEDVATEGPGLVFVVYPAAIATMPASTFWALIFFMMLLTI

TMHMM PROTTER S6A14_HUMAN DAT_DROME

420 GLDSQFASIETITTTIQDLFPKVMKKMRVP ITLGCCLVLFLLGLVCVTQA 418 GLDSSEGGSEAIITALSDEFPKI-KRNRELFVAGLFSLYFVVGLASCTQG 


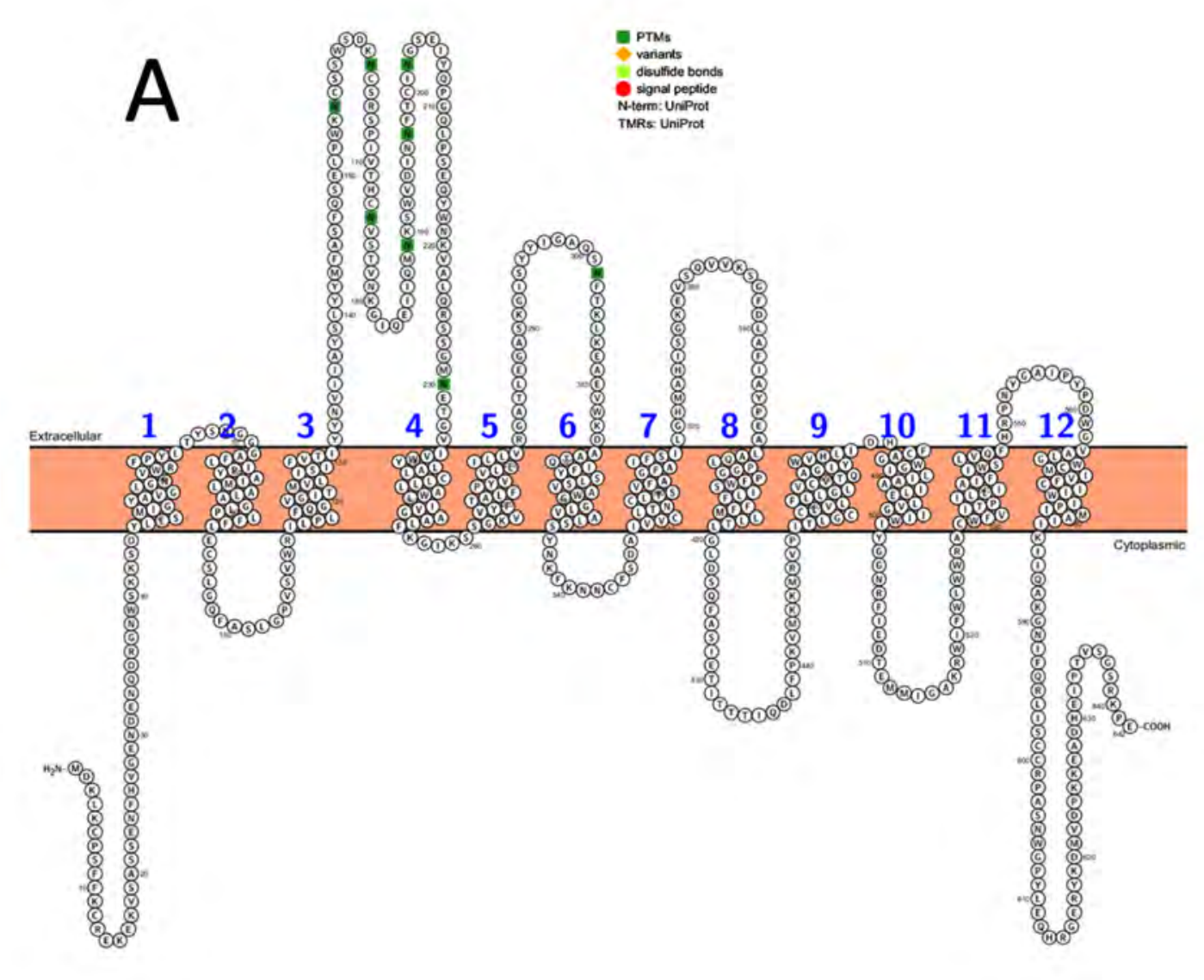

B

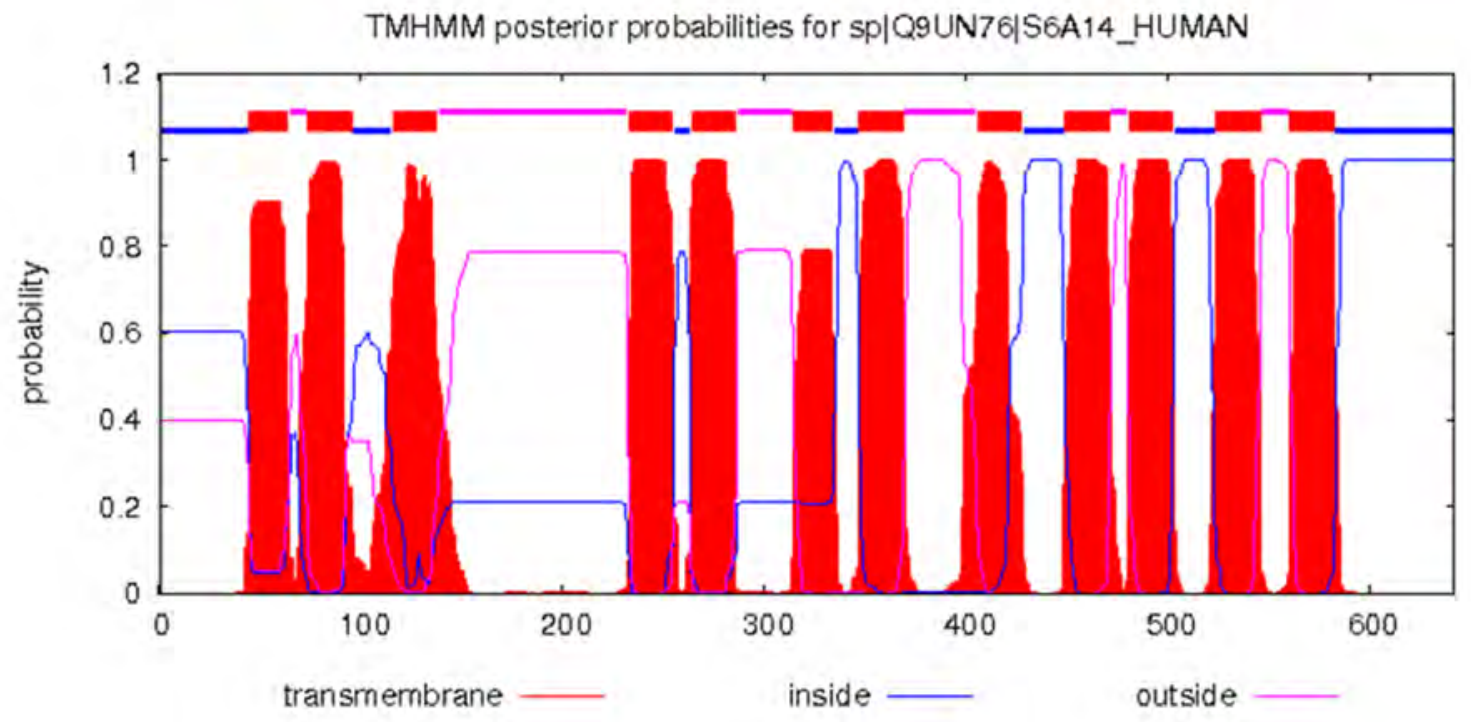


A

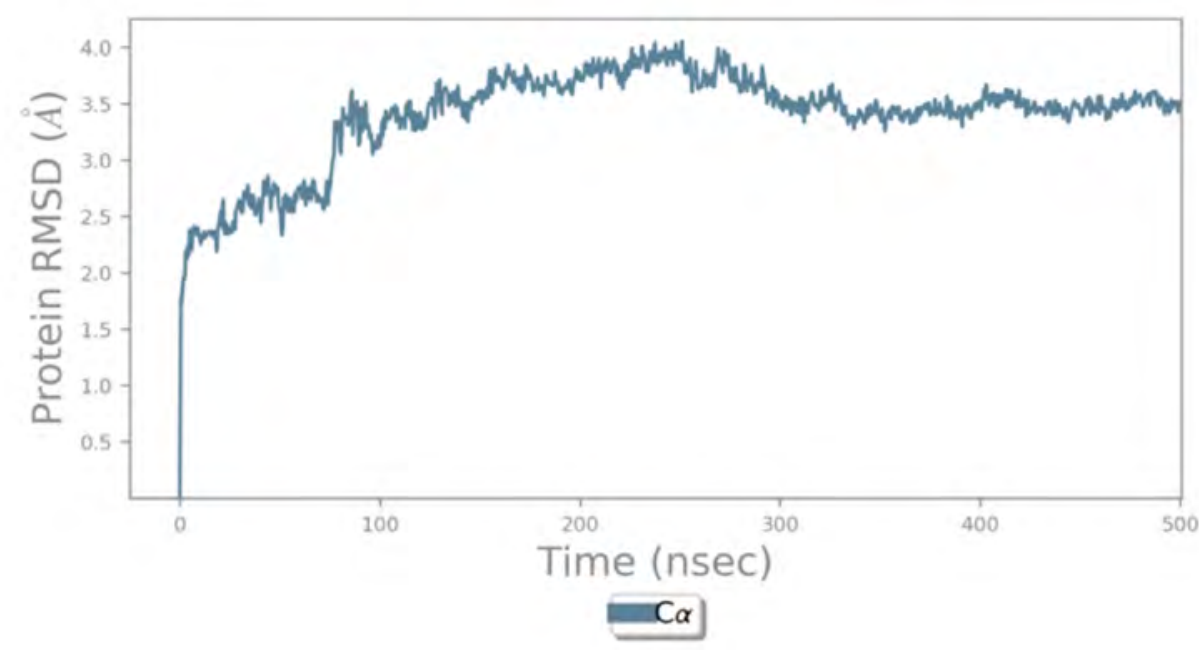

B

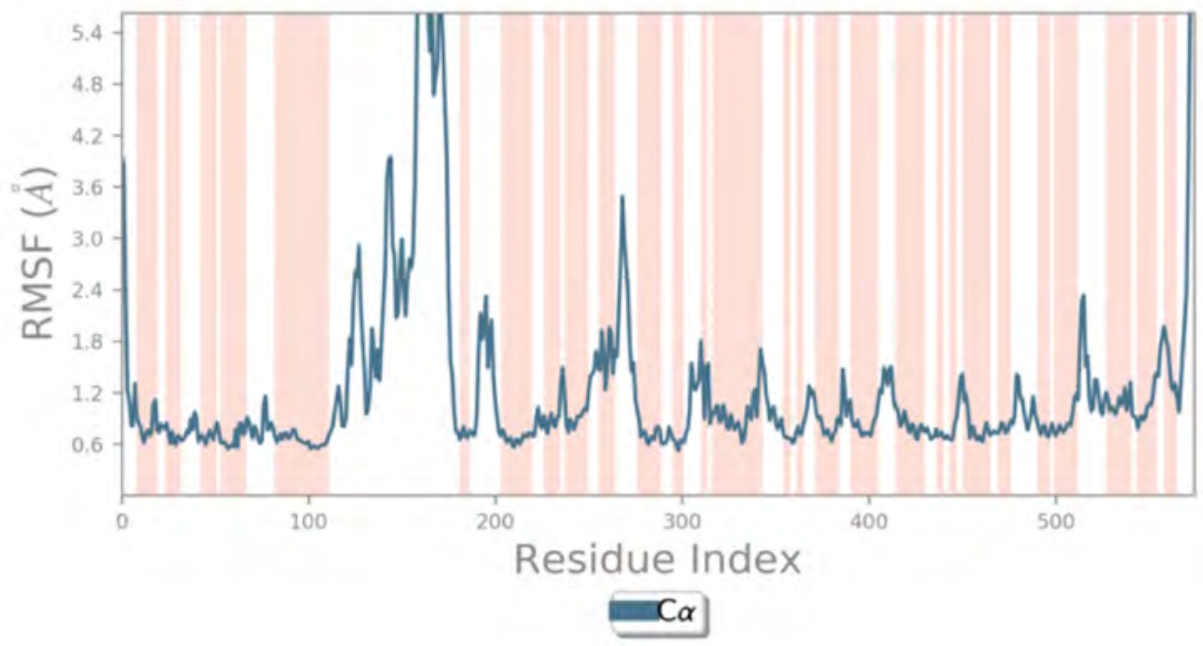

C

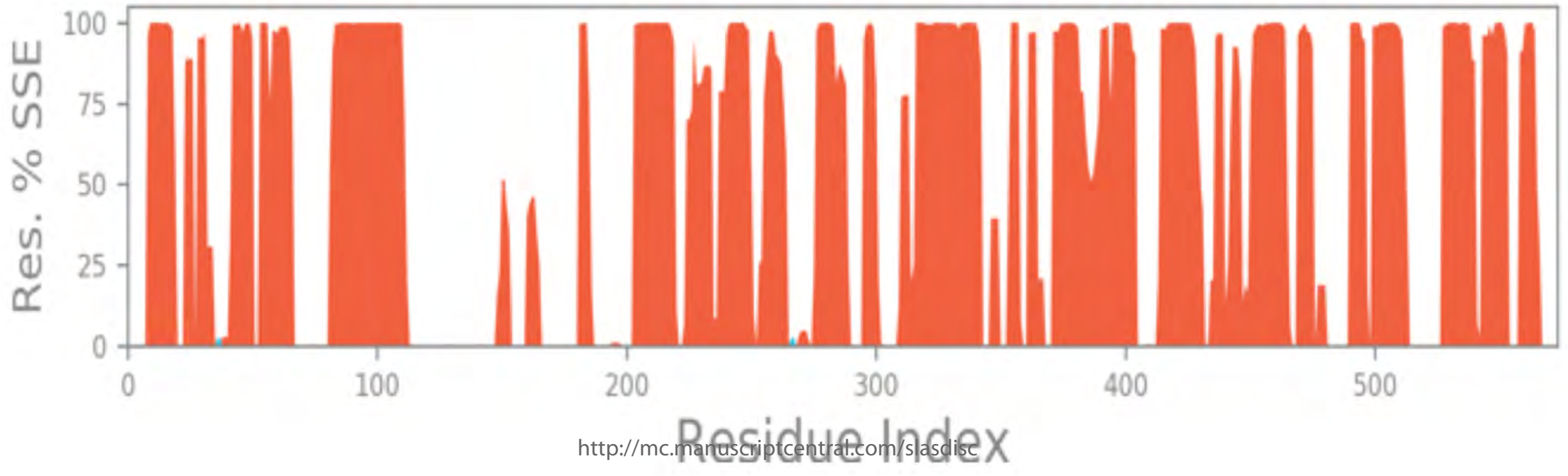



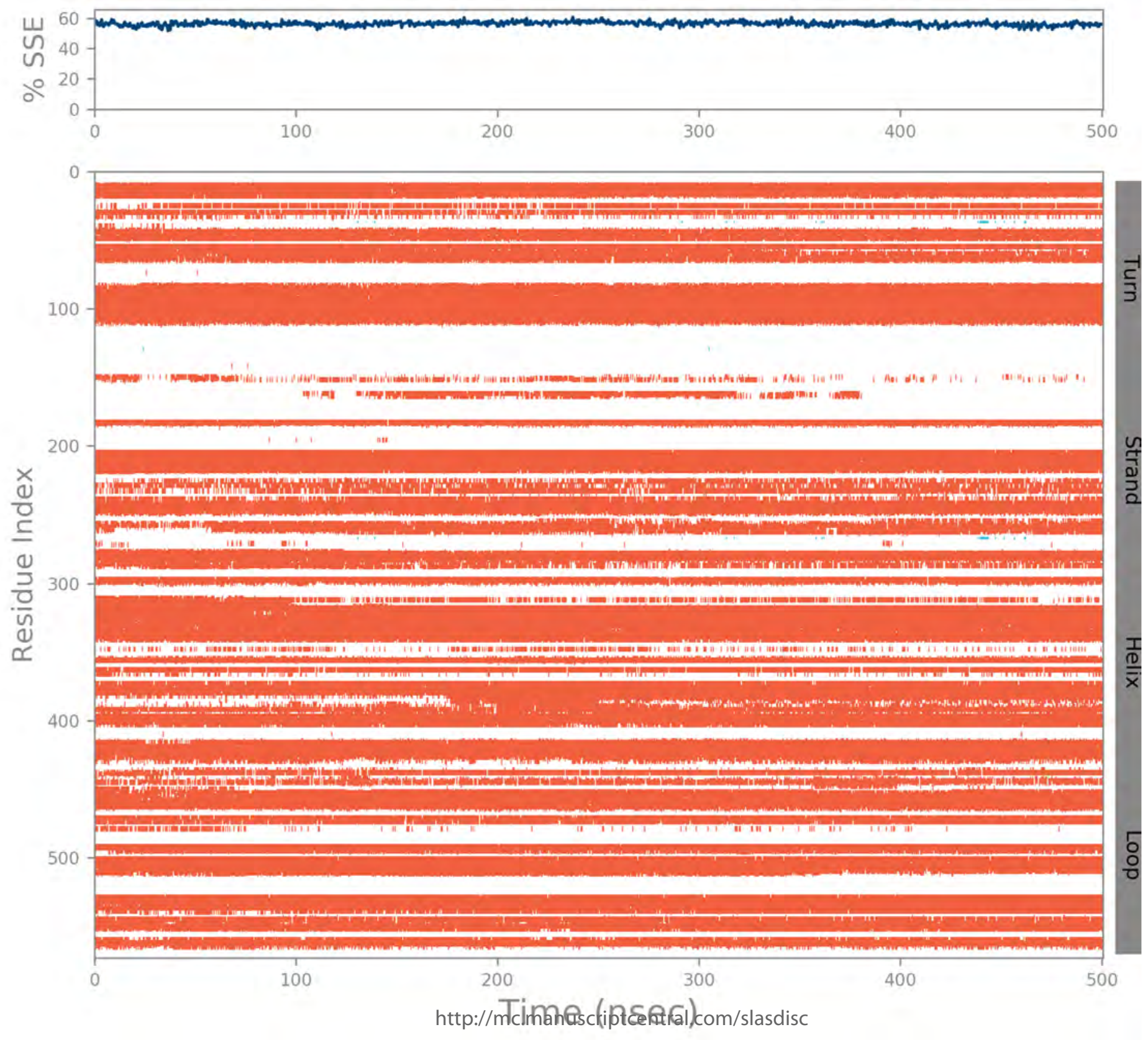

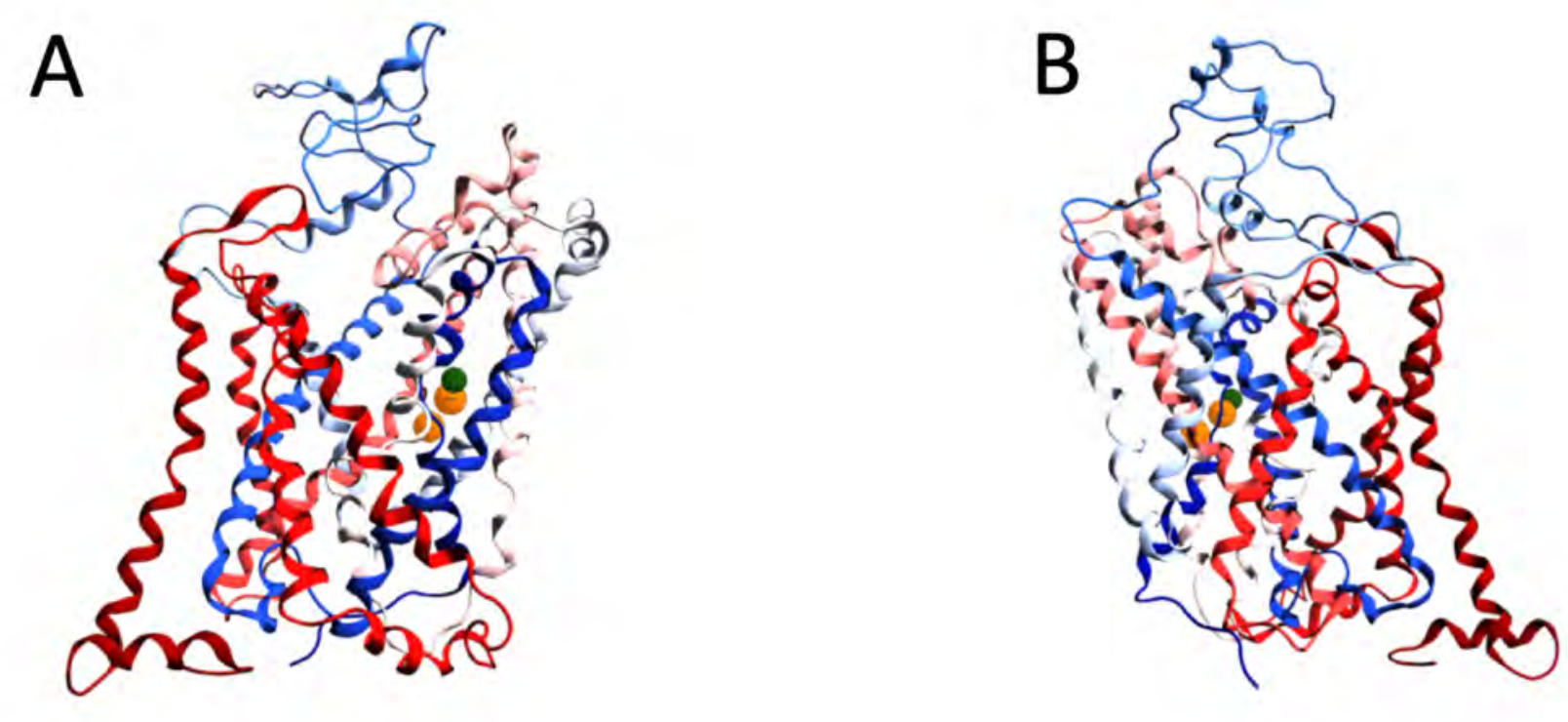

C

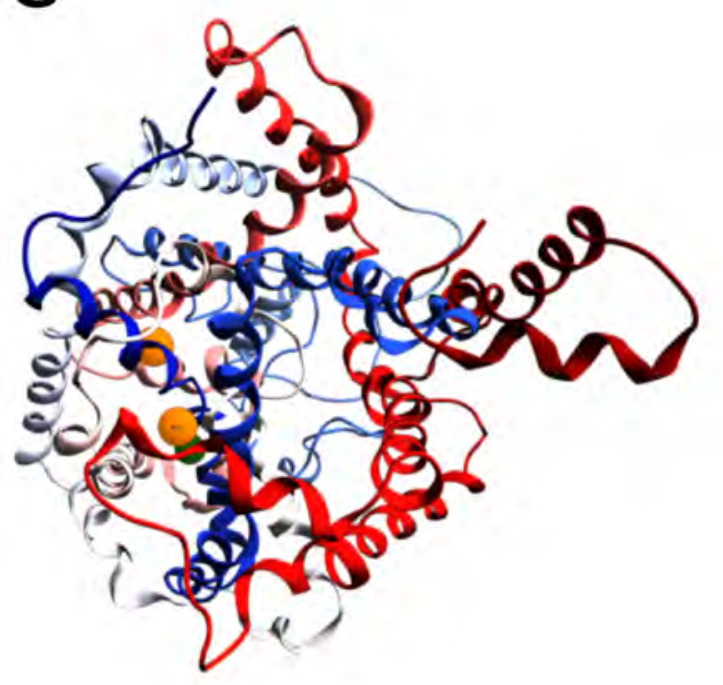

D

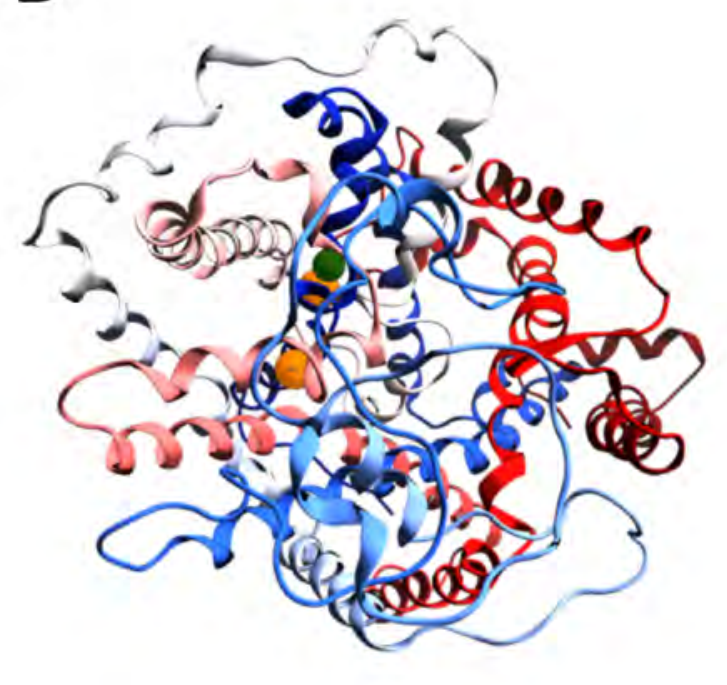




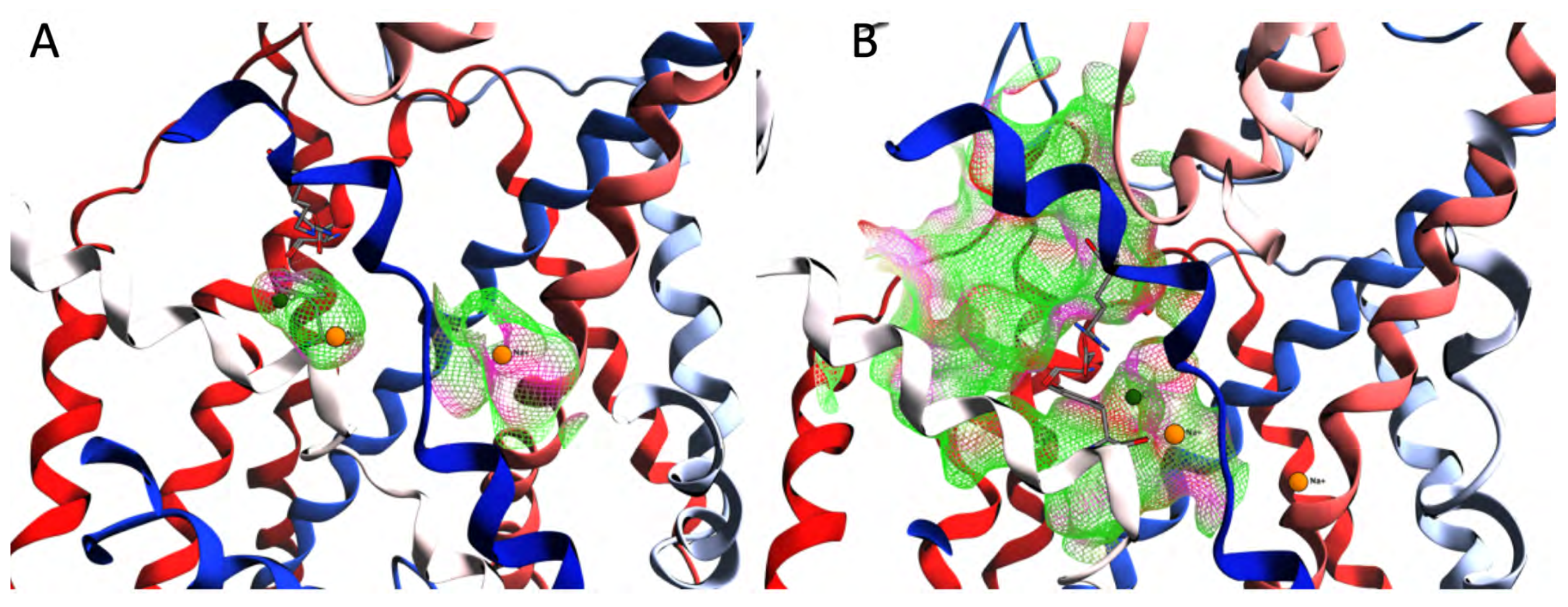


A

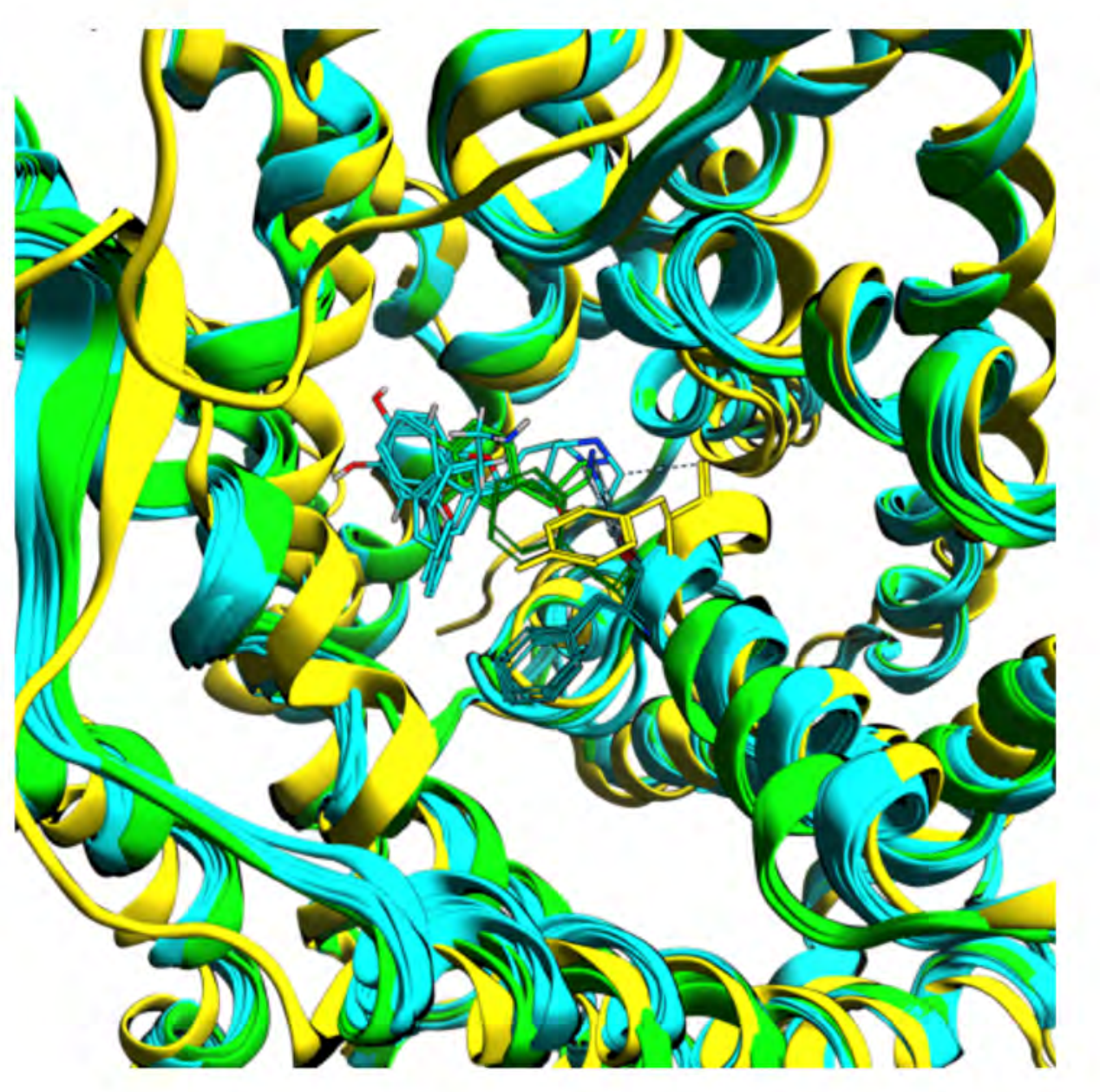

B

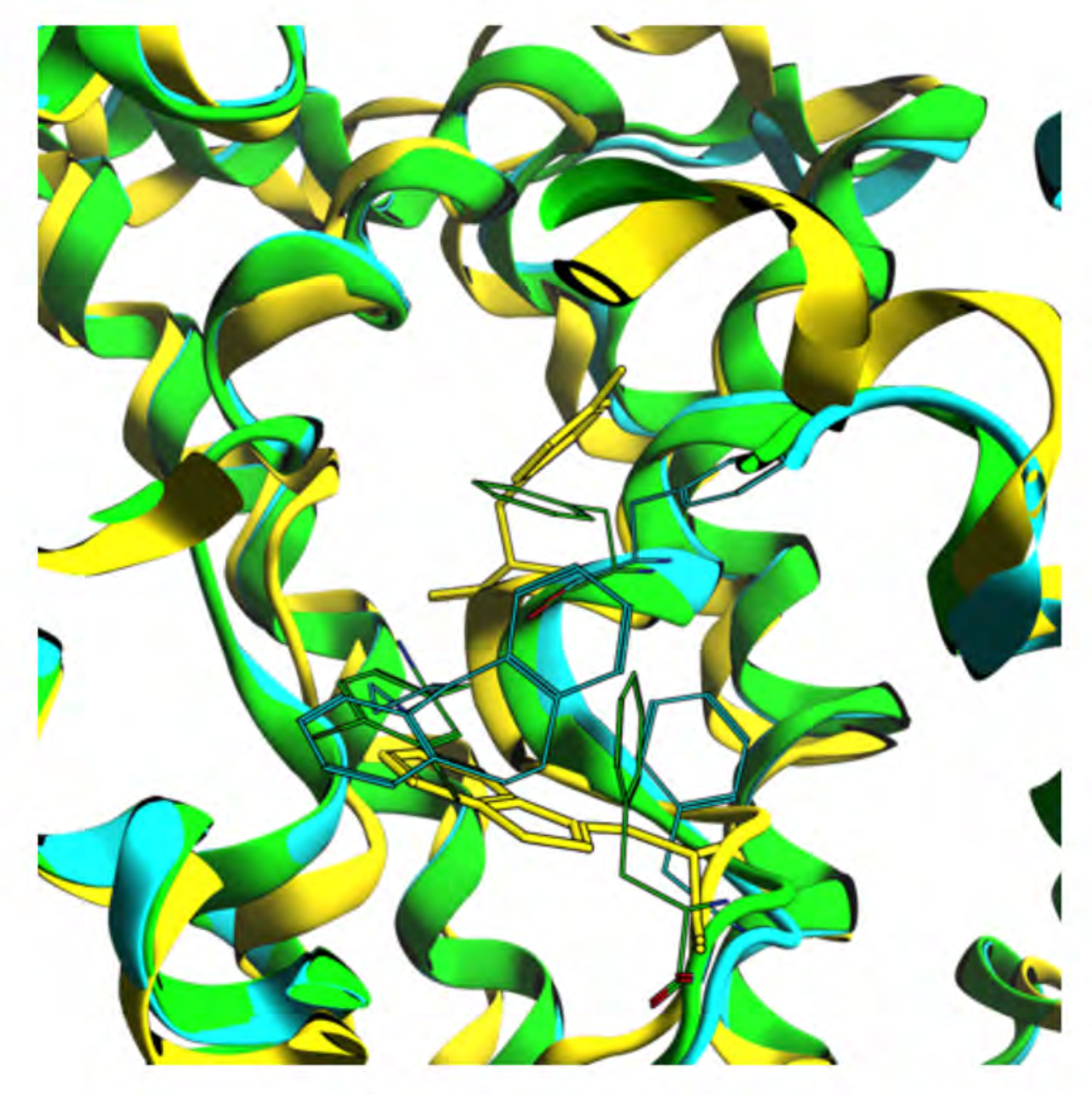




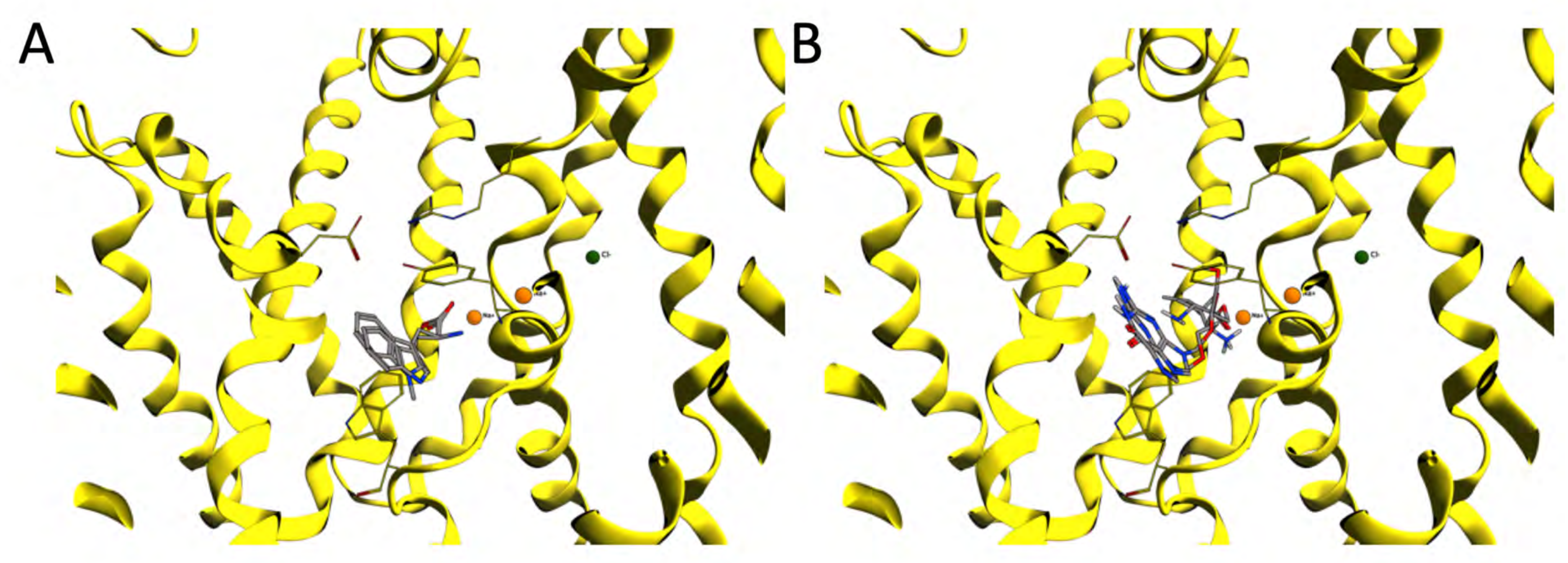



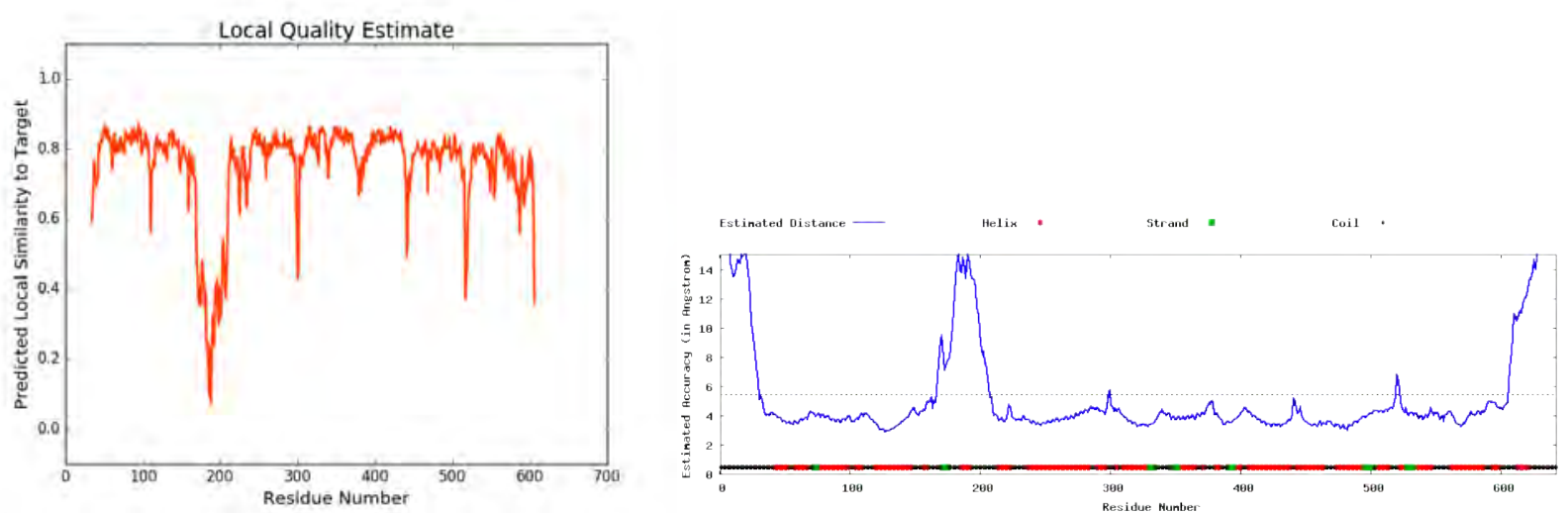

Supplementary Figure 1: (A) Local quality estimation of Swiss Model results and (B) I-Tasser estimated distance between model and theoretical folding.

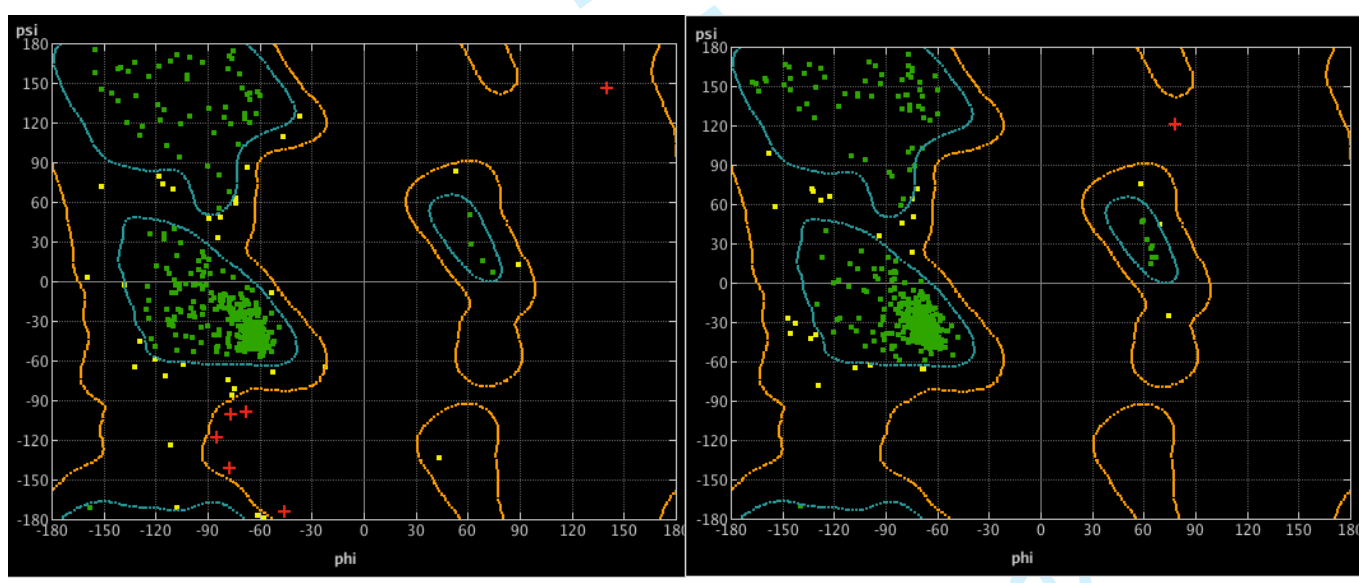

Supplementary Figure 2: Ramachandran plot of SLC6A14 chimera model before (A) and after (B) the molecular dynamics equilibration. 

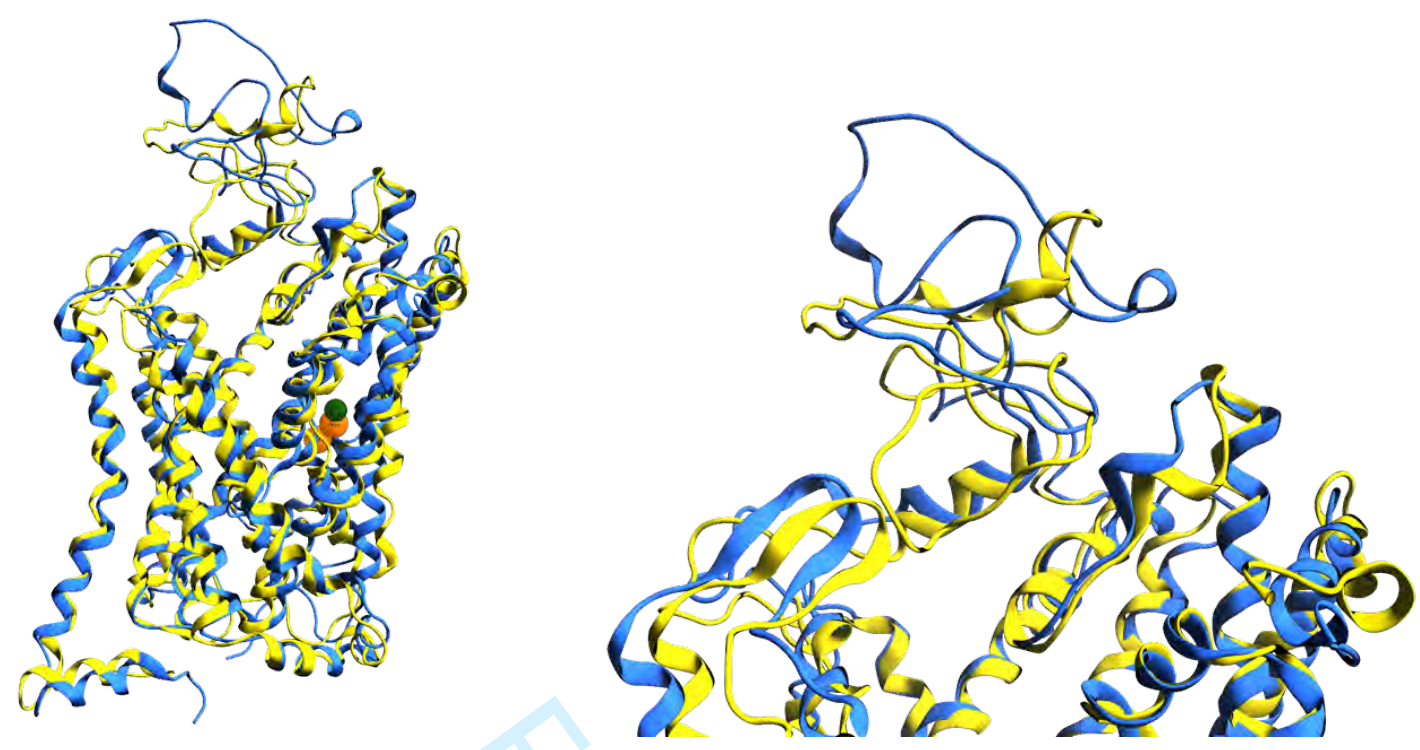

Supplementary Figure 3: Superposition of SLC6A14 conformations (A) with a focus on EL2 (B). In yellow is depicted the SLC6A14 structure from homology modelling procedure, before to be submitted to $500 \mathrm{~ns}$ MD equilibration; in blue the centroid of the most populated cluster. It is evident the structural rearrangement of EL2. 


\begin{tabular}{|c|c|c|c|c|c|c|}
\hline & Arg & Gln & Gly & 1-met-Trp & Phe & Trp \\
\hline \multicolumn{7}{|l|}{ Gly 51} \\
\hline Tyr 52 & & WB & WB & $\mathrm{H}$ & WB & $\mathrm{H}$ \\
\hline Ala 53 & & 1 & & 1 & 1 & $\mathrm{HB}$ \\
\hline Val 54 & & & WB & & & \\
\hline Gly 55 & & 1 & & 1 & 1 & $\mathrm{HB}$ \\
\hline Leu 56 & & WB & $\mathrm{H}$ & $\mathrm{HB}$ & $\mathrm{HB}$ & $\mathrm{HB}$ \\
\hline Gly 57 & I & & $\mathrm{H}$ & WB & $\mathrm{HB}$ & $\mathrm{HB}$ \\
\hline Asn 58 & & & & & 1 & 1 \\
\hline Trp 60 & $\mathrm{H}$ & & & & $\mathrm{H}$ & \\
\hline Arg 61 & & & WB & & WB & \\
\hline Ile 124 & & & & $\mathrm{H}$ & & \\
\hline Val 128 & $\mathrm{H}$ & $\mathrm{H}$ & & $\mathrm{H}$ & $\mathrm{H}$ & \\
\hline Tyr 131 & WB & WB & & & $\mathrm{HB}$ & \\
\hline Tyr 132 & $\mathrm{HB}$ & $\mathrm{HB}$ & $\mathrm{HB}$ & $\mathrm{HB}$ & $\mathrm{H}$ & WB \\
\hline Ile 135 & & 2 & & & & \\
\hline Thr 317 & WB & WB & & & & \\
\hline$G \ln 318$ & & 3 & & & WB & \\
\hline Phe 320 & $\mathrm{HB}$ & $\mathrm{HB}$ & WB & $\mathrm{HB}$ & WB & $\mathrm{HB}$ \\
\hline Tyr 321 & $\mathrm{H}$ & 1 & & $\mathrm{H}$ & $\mathrm{H}$ & 1 \\
\hline Ser 322 & & 1 & WB & 1 & 1 & 1 \\
\hline Ser 324 & $\mathrm{HB}$ & $i$ & $\mathrm{H}$ & $\mathrm{HB}$ & 1 & $\mathrm{HB}$ \\
\hline Val 325 & $\mathrm{HB}$ & WB & 10 & & & \\
\hline Ala 326 & $\mathrm{HB}$ & WB & 2 & & & \\
\hline Trp 327 & $\mathrm{H}$ & $\mathrm{H}$ & & $\mathrm{H}$ & $\mathrm{H}$ & $\mathrm{H}$ \\
\hline Asn 354 & & & 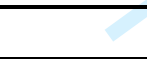 & 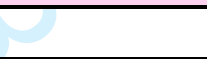 & & 1 \\
\hline Phe 388 & & & & 40 & $\mathrm{H}$ & \\
\hline Leu 419 & & & WB & 8 & & \\
\hline Gly 420 & & & WB & 80 & & \\
\hline \multicolumn{7}{|l|}{ Asp 422} \\
\hline Ser 423 & & WB & WB & WB & WB & WB \\
\hline$G \ln 424$ & & & WB & $E$ & & \\
\hline Ile 477 & WB & & & & & \\
\hline Asp 478 & $\mathrm{HB}$ & $\mathrm{HB}$ & & & WB & \\
\hline Cys 481 & WB & $\mathrm{HB}$ & & & & \\
\hline Ala 482 & WB & & & $\mathrm{H}$ & & \\
\hline Ile 486 & $\mathrm{H}$ & & & $\mathrm{H}$ & & $\mathrm{H}$ \\
\hline
\end{tabular}

$\begin{array}{cl}\text { I } & \text { lonic } \\ \text { HB } & \text { H-bond } \\ \text { H } & \text { Hydrophobic } \\ \text { WB } & \text { Water Bridge }\end{array}$




\begin{tabular}{|c|c|c|c|c|c|c|}
\hline & Tyr & valaciclovir & valganciclovir & Leu & His & Pro \\
\hline Gly 51 & & $\mathrm{HB}$ & & & & \\
\hline Tyr 52 & $\mathrm{H}$ & $\mathrm{H}$ & $\mathrm{H}$ & $\mathrm{H}$ & $\mathrm{H}$ & WB \\
\hline Ala 53 & & & & 1 & $\mathrm{H}$ & 1 \\
\hline Val 54 & & $\mathrm{HB}$ & & 1 & & \\
\hline Gly 55 & & & & & & WB \\
\hline Leu 56 & $\mathrm{HB}$ & $\mathrm{HB}$ & $\mathrm{HB}$ & $\mathrm{HB}$ & & \\
\hline Gly 57 & $\mathrm{HB}$ & & WB & $\mathrm{HB}$ & $\mathrm{HB}$ & \\
\hline Asn 58 & & & & & 1 & \\
\hline Trp 60 & & & WB & $\mathrm{H}$ & & \\
\hline $\operatorname{Arg} 61$ & & WB & $\mathrm{H}$ & & $\mathrm{HB}$ & WB \\
\hline \multicolumn{7}{|l|}{ Ile 124} \\
\hline Val 128 & $\mathrm{H}$ & $\mathrm{H}$ & $\mathrm{H}$ & $\mathrm{H}$ & $\mathrm{H}$ & \\
\hline Tyr 131 & & WB & WB & $\mathrm{H}$ & & \\
\hline Tyr 132 & $\mathrm{H}$ & $\mathrm{HB}$ & $\mathrm{HB}$ & $\mathrm{HB}$ & $\mathrm{HB}$ & $\mathrm{HB}$ \\
\hline Ile 135 & & 2 & & $\mathrm{H}$ & & \\
\hline Thr 317 & & WB & & & & \\
\hline \multicolumn{7}{|l|}{$G \ln 318$} \\
\hline Phe 320 & $\mathrm{H}$ & WB & WB & WB & $\mathrm{HB}$ & $\mathrm{HB}$ \\
\hline Tyr 321 & $\mathrm{H}$ & $\mathrm{H}$ & $\mathrm{H}$ & $\mathrm{H}$ & 1 & 1 \\
\hline Ser 322 & & & & 1 & 1 & \\
\hline Ser 324 & $\mathrm{HB}$ & $\mathrm{HB}$ & $\mathrm{HB}$ & WB & 1 & $\mathrm{HB}$ \\
\hline Val 325 & WB & WB & $\mathrm{HB}$ & & & $\mathrm{HB}$ \\
\hline Ala 326 & WB & & 8 & & $\mathrm{HB}$ & \\
\hline Trp 327 & $\mathrm{H}$ & $\mathrm{H}$ & $\mathrm{H}$ & $\mathrm{H}$ & $\mathrm{H}$ & $\mathrm{H}$ \\
\hline Asn 354 & & & $P$ & P & & \\
\hline \multicolumn{7}{|l|}{ Phe 388} \\
\hline Leu 419 & & & $\mathrm{HB}$ & 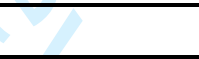 & & \\
\hline Gly 420 & WB & & $\mathrm{HB}$ & 20 & & \\
\hline Asp 422 & $\mathrm{H}$ & $\mathrm{HB}$ & $\mathrm{HB}$ & 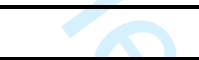 & & \\
\hline Ser 423 & $\mathrm{HB}$ & & $\mathrm{HB}$ & 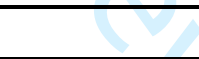 & & WB \\
\hline$G \ln 424$ & WB & & & $E$ & & \\
\hline Ile 477 & & $\mathrm{HB}$ & $\mathrm{HB}$ & & & \\
\hline Asp 478 & WB & $\mathrm{HB}$ & $\mathrm{HB}$ & & WB & \\
\hline Cys 481 & WB & $\mathrm{HB}$ & $\mathrm{HB}$ & & & \\
\hline Ala 482 & & $\mathrm{HB}$ & $\mathrm{HB}$ & & WB & $\mathrm{H}$ \\
\hline Ile 486 & & $\mathrm{H}$ & $\mathrm{H}$ & & $\mathrm{H}$ & $\mathrm{H}$ \\
\hline
\end{tabular}

Ionic

HB H-bond

H Hydrophobic

WB Water Bridge 


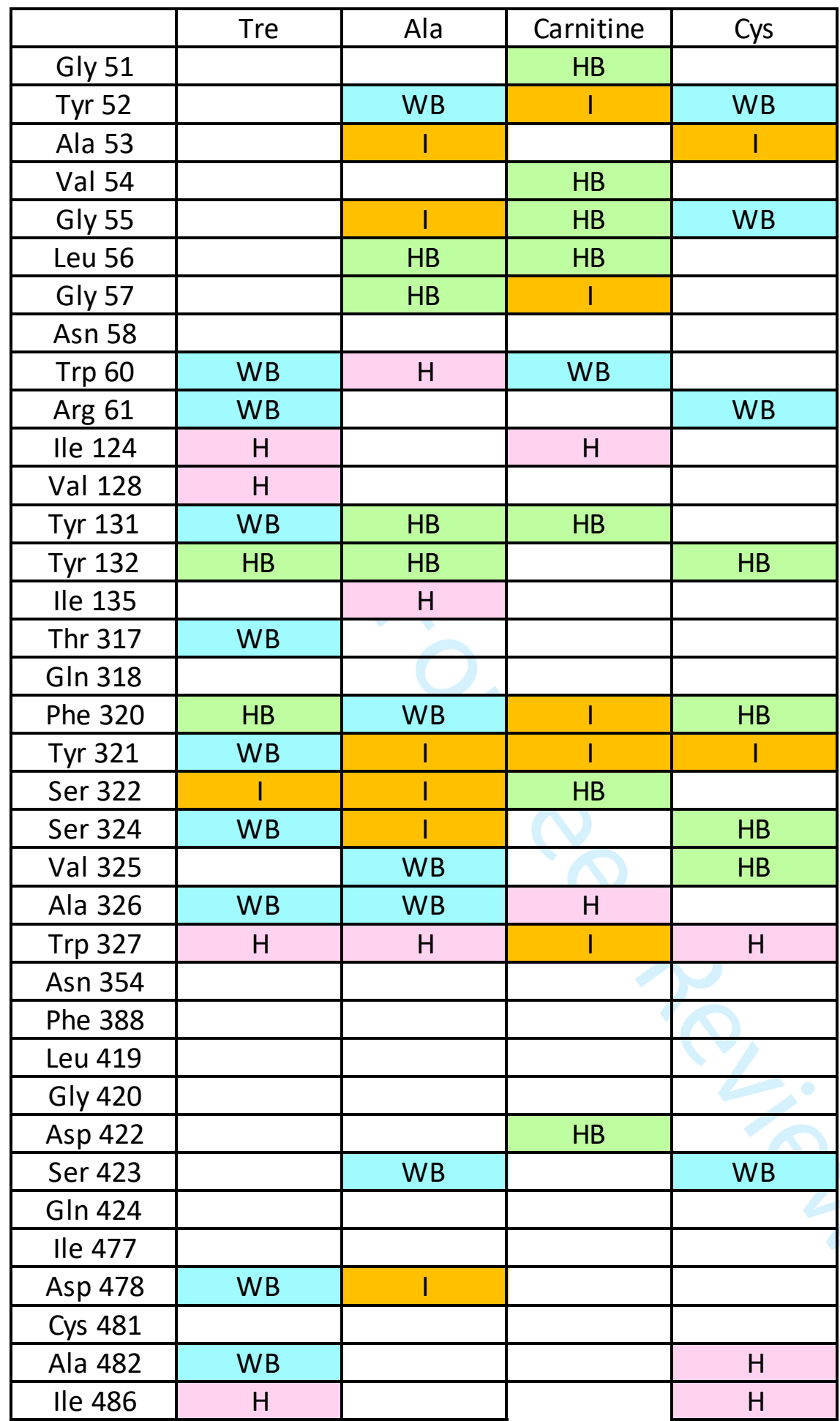

\begin{tabular}{cl}
\hline I & lonic \\
\hline HB & H-bond \\
H & Hydrophobic \\
WB & Water Bridge
\end{tabular}

Supplementary Table 1 